\title{
Scrutinizing vacuum stability in IDM with Type-III inverse seesaw
}

\author{
Priyotosh Bandyopadhyay, ${ }^{a}$ Shilpa Jangid ${ }^{a}$ and Manimala Mitra ${ }^{b, c}$ \\ ${ }^{a}$ Indian Institute of Technology Hyderabad, \\ Kandi, Sangareddy-502287, Telangana, India \\ ${ }^{b}$ Institute of Physics Bhubaneswar, \\ Sachivalaya Marg, Bhubaneswar 751005, India \\ ${ }^{c}$ Homi Bhabha National Institute, BARC Training School Complex, \\ Anushakti Nagar, Mumbai 400094, India \\ E-mail: bpriyo@phy.iith.ac.in, ph19resch02006@iith.ac.in, \\ manimala@iopb.res.in
}

AbStract: We consider the extension of the Standard Model (SM) with an inert Higgs doublet that also contains two or three sets of $\mathrm{SU}(2)_{L}$ triplet fermions with hypercharge zero and analyze the stability of electroweak vacuum for the scenarios. The model represents a Type-III inverse seesaw mechanism for neutrino mass generation with a Dark matter candidate. An effective potential approach calculation with two-loop beta function have been carried out in deciding the fate of the electroweak vacuum. Weak gauge coupling $g_{2}$ shows a different behaviour as compared to the Standard Model. The modified running of $g_{2}$, along with the Higgs quartic coupling and Type-III Yukawa couplings become crucial in determining the stability of electroweak vacuum. The interplay between two and three generations of such triplet fermions reveals that extensions with two generations is favoured if we aspire for Planck scale stability. Bounds on the Higgs quartic couplings, Type-III Yukawa and number of triplet fermion generations are drawn for different mass scale of Type-III fermions. The phenomenologies of inert doublet and Type-III fermions at the LHC and other experiments are commented upon.

Keywords: Beyond Standard Model, Higgs Physics, Neutrino Physics

ARXIV EPRINT: 2008.11956 


\section{Contents}

1 Introduction 1

2 The model $\quad 2$

2.1 The scalar sector 3

2.2 The Type-III and inverse seesaw Lagrangians 4

3 Perturbativity $\quad 6$

$\begin{array}{ll}3.1 \text { Running of gauge couplings } & 6\end{array}$

4 Stability bound $\quad 13$

$\begin{array}{lll}\text { 4.1 RG evolution of the scalar quartic couplings } & 13\end{array}$

$\begin{array}{lll}4.2 \text { Variation perturbativity and stability with } Y_{N} & 16\end{array}$

$\begin{array}{ll}4.3 \text { Vacuum stability: RG-improved potential approach } & 17\end{array}$

$\begin{array}{lll}4.4 & \text { Effective potential } & 18\end{array}$

$\begin{array}{ll}\text { 4.5 Phase diagrams: stable, metastable and unstable regions } & 19\end{array}$

5 Discussions and conclusion $\quad 23$

A Two-loop $\beta$-functions with two generations $\quad 24$

A.1 Scalar quartic couplings 24

$\begin{array}{lll}\text { A.2 Yukawa coupling } & 27\end{array}$

\section{Introduction}

The last element of the Standard Model (SM) was the Higgs boson which was discovered at the CMS and ATLAS detectors of the Large Hadron Collider (LHC) [1, 2]. The spin, parity measurements and the combined analysis show the SM-like behaviour of the Higgs boson [3-5]. However it has been shown that Standard Model electroweak (EW) vacuum on its own can run into metastability due to quantum corrections [6-9]. It is well known that the addition of scalars enhance the stability of the EW vacuum via positive loop contributions to the Higgs quartic coupling. Various models, which include scalars from different gauge representations have been proposed [10-53] to enhance the stability of EW vacuum. On the contrary an extension with fermion often gives negative contributions to the Higgs quartic couplings that it couples to. Such negative contributions then tend to pull such Higgs quartic couplings toward instability much faster. Thus models with extra fermions and where Majorana masses of the fermions are spontaneously generated are constrained from the vacuum stability [54-76].

Apart from the problem of vacuum metastability in SM which depends on the top quark and Higgs boson masses [77-79], the theory also fails to provide a stable dark matter 
(DM) candidate, as well as to give successful explanation for the very tiny eV scale neutrino masses, and their mixings. In this work, we focus on these two aspects by extending the $\mathrm{SM}$ with $\mathrm{SU}(2)_{L}$ triplet fermions, and $\mathrm{SU}(2)_{L}$ inert doublet scalar. The triplet fermion generates the $\mathrm{eV}$ light neutrino mass via Type-III seesaw mechanism, while the inert Higgs doublet provides a dark matter candidate, as well as stabilizes the EW vacuum.

The minimal Type-III extensions have one to three generations of $\mathrm{SU}(2)_{L}$ fermions with hypercharge zero, which mix with the SM charged and neutral fermions, and also generates tiny eV neutrino mass via electroweak symmetry breaking [80-83]. Different extensions of Type-III seesaw and their collider signatures have been studied in [84]-[90], including their spin measurement at the LHC [91]. The stability of EW vacuum in some of these scenarios are studied in [92-95]. In this article, we consider the inverse seesaw mechanism of neutrino mass generation with two generations of triplet fermions. One among them couples with the SM Higgs boson via Type-III Yukawa coupling, and generates the Dirac mass term. The other triplet fermion generates the Majorana mass term for the triplet fermion.

As discussed earlier an extension with scalar enhances stability of EW vacuum and if the scalar is in the form of $\mathrm{SU}(2)_{L}$ inert $\left(Z_{2}\right.$-odd) doublet then it also provides the much needed dark matter candidate [34-36]. SM extension with such inert doublet in the context of vacuum stability have been studied extensively [34-39, 54]. Fields in inert doublet have very interesting phenomenology due to their compressed spectrum and the possibility of real and pseudoscalar dark matter particle [96]-[107]. In this article we will investigate the effect of inert doublet in the context of Type-III fermions.

In our model we have both $\mathrm{SU}(2)_{L}$ triplet fermion and $\mathrm{SU}(2)_{L}$ doublet scalar. Being in the triplet representation of $\mathrm{SU}(2)_{L}$ the new fermions contribute in the evolution of weak gauge coupling $g_{2}$ such that $g_{2}$ now increases with running scale. This behaviour substantially changes the dynamics of couplings responsible for the EW vacuum stability. We shall see how an enhanced $g_{2}$ causes a much lower perturbative scale compared to Type-I [54, 55] or only IDM case [38]. Specially we see that with three generations of TypeIII fermions it is difficult to attain Planck scale perturbativity and thus two generations are more favoured.

The paper is organised as follows. In section 2 , we describe the model, and present the EW symmetry breaking conditions for this model. We discuss the perturbativity and the interplay of two and three generations of $\mathrm{SU}(2)_{L}$ triplet fermions in section 3 . The EW vacuum stability with all three posibilites are covered in section 4 . In section 5 , we discuss the phenomenological consequences and present our conclusion. The expressions of the two-loop beta functions used in our analysis are presented in appendix A.

\section{The model}

The SM is augmented with an inert doublet (ID) and three Type-III fermions with ISS mechanism. We first discuss the scalar sector of the model in section 2.1. We consider two different scenarios, viz., a canonical Type-III seesaw with small Yukawa couplings and an inverse seesaw (ISS) with large Yukawa couplings to study effects in perturbativity of dimensionless couplings and vacuum stability. The fermionic sector with Type-III seesaw and inverse seesaw is discussed in section 2.2 . 


\subsection{The scalar sector}

The scalar part consists of two $\mathrm{SU}(2)_{L}$-doublets $\Phi_{1}$ and $\Phi_{2}$, both with hypercharge $1 / 2$ :

$$
\Phi_{1}=\left(\begin{array}{c}
G^{+} \\
h+i G^{0}
\end{array}\right), \quad \Phi_{2}=\left(\begin{array}{c}
H^{+} \\
H+i A
\end{array}\right) .
$$

The tree-level SM gauge invariant scalar potential i.e., invariant under $\mathrm{SU}(2)_{L} \times \mathrm{U}(1)_{Y}$ is given by $[108,109]$

$$
\begin{aligned}
V_{\text {scalar }}= & m_{11}^{2} \Phi_{1}^{\dagger} \Phi_{1}+m_{22}^{2} \Phi_{2}^{\dagger} \Phi_{2}-\left(m_{12}^{2} \Phi_{1}^{\dagger} \Phi_{2}+\text { H.c }\right) \\
& +\lambda_{1}\left(\Phi_{1}^{\dagger} \Phi_{1}\right)^{2}+\lambda_{2}\left(\Phi_{2}^{\dagger} \Phi_{2}\right)^{2}+\lambda_{3}\left(\Phi_{1}^{\dagger} \Phi_{1}\right)\left(\Phi_{2}^{\dagger} \Phi_{2}\right)+\lambda_{4}\left(\Phi_{1}^{\dagger} \Phi_{2}\right)\left(\Phi_{2}^{\dagger} \Phi_{1}\right) \\
& +\left[\lambda_{5}\left(\Phi_{1}^{\dagger} \Phi_{2}\right)^{2}+\lambda_{6}\left(\Phi_{1}^{\dagger} \Phi_{1}\right)\left(\Phi_{1}^{\dagger} \Phi_{2}\right)+\lambda_{7}\left(\Phi_{2}^{\dagger} \Phi_{2}\right)\left(\Phi_{1}^{\dagger} \Phi_{2}\right)+\text { H.c }\right],
\end{aligned}
$$

where we have chosen all the Higgs quartic couplings $\lambda_{1,2,3,4}$ and mass terms $m_{11}^{2}, m_{22}^{2}$ as real. While $m_{12}^{2}$ and the $\lambda_{5,6,7}$ couplings are in general complex but for this study we also have taken them as real number. A $Z_{2}$ symmetry is imposed to prohibit the flavor changing neutral currents at tree-level. Under this $Z_{2}$ symmetry $\Phi_{2}$ is odd and $\Phi_{1}$ is even. This choice also make $\Phi_{2}$ as inert which can be DM candidate. The $\lambda_{6}, \lambda_{7}$ and $m_{12}$ also get removed and eq. (2.2) reduces to

$$
\begin{aligned}
V_{\text {scalar }}= & m_{11}^{2} \Phi_{1}^{\dagger} \Phi_{1}+m_{22}^{2} \Phi_{2}^{\dagger} \Phi_{2}+\lambda_{1}\left(\Phi_{1}^{\dagger} \Phi_{1}\right)^{2}+\lambda_{2}\left(\Phi_{2}^{\dagger} \Phi_{2}\right)^{2} \\
& +\lambda_{3}\left(\Phi_{1}^{\dagger} \Phi_{1}\right)\left(\Phi_{2}^{\dagger} \Phi_{2}\right)+\lambda_{4}\left(\Phi_{1}^{\dagger} \Phi_{2}\right)\left(\Phi_{2}^{\dagger} \Phi_{1}\right)+\left[\lambda_{5}\left(\Phi_{1}^{\dagger} \Phi_{2}\right)^{2}+\text { H.c }\right] .
\end{aligned}
$$

The $\Phi_{1}$ takes a real vacuum expectation value (VEV) which breaks the EW symmetry as follows

$$
\left\langle\Phi_{1}\right\rangle=\frac{1}{\sqrt{2}}\left(\begin{array}{l}
0 \\
v
\end{array}\right),
$$

here we choose $v \simeq 246 \mathrm{GeV}$ guided by the masses of gauge bosons and SM-like Higgs boson. $\Phi_{2}$, being $Z_{2}$-odd, does not have any part in breaking the EW symmetry, so it behaves like a 'inert' Higgs doublet. Such model is often named as inert doublet model (IDM). We replace $m_{11}^{2}$ by the minimzation condition follows:

$$
m_{11}^{2}=-\lambda_{1} v^{2}
$$

where the respective physical mass eigenvalues can be written as

$$
\begin{aligned}
M_{h}^{2} & =2 \lambda_{1} v^{2}, \\
M_{H_{0}}^{2} & =\frac{1}{2}\left[2 m_{22}^{2}+v^{2}\left(\lambda_{3}+\lambda_{4}+2 \lambda_{5}\right)\right], \\
M_{A}^{2} & =\frac{1}{2}\left[2 m_{22}^{2}+v^{2}\left(\lambda_{3}+\lambda_{4}-2 \lambda_{5}\right)\right], \\
M_{H^{ \pm}}^{2} & =m_{22}^{2}+\frac{1}{2} v^{2} \lambda_{3} .
\end{aligned}
$$

This is to note that, being $Z_{2}$ odd $\Phi_{2}$ is inert, which prohibits any mixing between $\Phi_{2}$ and $\Phi_{1}$. This also implies that the gauge eigenstates and the mass eigenstates are the same for 
the Higgs bosons from both $Z_{2}$ odd or even multiplets. In this scenario, $\Phi_{2}$ being $Z_{2}$-odd does not talk to the fermions. Moreover, we get two CP even neutral Higgs bosons $h$ and $H_{0}$. Here we choose $h$ as the SM-like Higgs boson with mass around $125 \mathrm{GeV}$ discovered at the LHC. The spectrum has one pseudoscalar Higgs boson $A$ and a pair of charged Higgs bosons $H^{ \pm}$. It is evident from eq. (2.6) that the heavy Higgs bosons $H_{0}, A$ and $H^{ \pm}$are from $\Phi_{2}$ so they are nearly degenerate in mass spectrum with possible splitting by the help of $\lambda_{5}$. The sign of $\lambda_{5}$ is crucial in making one of the scalars between $A$ and $H_{0}$ as the lightest, and possible DM candidate. The $Z_{2}$-odd symmetry prohibits some of the decays of $\Phi_{2}$-type Higgs bosons...

\subsection{The Type-III and inverse seesaw Lagrangians}

In addition to the SM particle contents, the Type-III seesaw model contains $\mathrm{SU}(2)_{L}$ fermionic triplets $\Sigma$ with zero hypercharge. Being in the adjoint representation of the $\mathrm{SU}(2)_{L}$ group, the Majorana mass term $M_{N}$ of such triplets is gauge invariant. In terms of the usual two-by two notation for triplets, the beyond SM interactions are described by the Lagrangian:

$$
\mathcal{L}_{\mathrm{III}}=\operatorname{Tr}[\bar{\Sigma} i \not D \Sigma]-\frac{1}{2} \operatorname{Tr}\left[\bar{\Sigma} M_{N} \Sigma^{c}+\overline{\Sigma^{c}} M_{N}^{*} \Sigma\right]-\sqrt{2}\left(\widetilde{\Phi_{1}^{\dagger}} \bar{\Sigma} Y_{N} L+\bar{L} Y_{N}^{\dagger} \Sigma \widetilde{\Phi_{1}}\right),
$$

where $L \equiv(\nu, \ell)_{L}$ corresponds to the SM lepton doublet, while $\widetilde{\Phi}_{1}=i \sigma_{2} \Phi_{1}^{\star}$ (with $\sigma_{2}$ is the second Pauli matrix), $\Sigma^{c} \equiv C \bar{\Sigma}^{T}$ for each fermionic triplet as shown below.

$$
\Sigma=\left(\begin{array}{cc}
\Sigma^{0} / \sqrt{2} & \Sigma^{+} \\
\Sigma^{-} & -\Sigma^{0} / \sqrt{2}
\end{array}\right), \quad \Sigma^{c}=\left(\begin{array}{cc}
\Sigma^{0 c} / \sqrt{2} & \Sigma^{-c} \\
\Sigma^{+c} & -\Sigma^{0 c} / \sqrt{2}
\end{array}\right)
$$

We drop the generation indices here, but this is to remind that there are three set of fermionic triplets for three leptonic doublets. The covariant derivative generates the coupling between the $W$ bosons and the triplet fermions and they are proportional to $g_{2}$ as shown below,

$$
\not D_{\mu}=\not \partial_{\mu}-i \sqrt{2} g_{2}\left(\begin{array}{cc}
W_{\mu}^{3} / \sqrt{2} & W_{\mu}^{+} \\
W_{\mu}^{-} & -W_{\mu}^{3} / \sqrt{2}
\end{array}\right) .
$$

Without loss of generality, we start from the basis, where $M_{N}$ is real and diagonal. In order to consider the mixing of fermionic triplets with the charged leptons, it is convenient to express the four degrees of freedom of each charged triplet in terms of a single Dirac spinor:

$$
\psi=\Sigma_{R}^{+c}+\Sigma_{R}^{-}
$$

On the other hand the neutral fermionic triplet components can be left in two-component notation, since they have only two degrees of freedom and mix with neutrinos, which are also described by two-component fields. This leads to the Lagrangian as follows

$$
\begin{aligned}
\mathcal{L}_{\text {III }}= & \bar{\psi} i \not \partial+\overline{\Sigma_{R}^{0}} i \not \partial \Sigma_{R}^{0}-\bar{\psi} M_{N} \psi-\left(\overline{\Sigma_{R}^{0}} \frac{M_{N}}{2} \Sigma_{R}^{0 c}+\text { h.c. }\right) \\
& +g\left(W_{\mu}^{+} \overline{\Sigma_{R}^{0}} \gamma_{\mu} P_{R} \psi+W_{\mu}^{+} \overline{\Sigma_{R}^{0 c}} \gamma_{\mu} P_{L} \psi+\text { h.c. }\right)-g W_{\mu}^{3} \bar{\psi} \gamma_{\mu} \psi \\
& -\left(\Phi^{0} \overline{\Sigma_{R}^{0}} Y_{N} \nu_{L}+\Phi^{+} \overline{\Sigma_{R}^{0}} Y_{N} \ell_{L}+\sqrt{2}\left(\Phi^{0} \bar{\psi} Y_{N} \ell_{L}-\Phi^{+} \overline{\nu_{L}^{c}} Y_{N}^{T} \psi\right)+\text { h.c. }\right) .
\end{aligned}
$$


The mass term of the charged sector shows the usual aspect for Dirac particles:

$$
\mathcal{L} \ni-\left(\overline{l_{R}} \overline{\psi_{R}}\right)\left(\begin{array}{cc}
m_{l} & 0 \\
Y_{N} v & M_{N}
\end{array}\right)\left(\begin{array}{c}
l_{L} \\
\psi_{L}
\end{array}\right)-\left(\overline{l_{L}} \overline{\psi_{L}}\right)\left(\begin{array}{cc}
m_{l} & Y_{N}^{\dagger} v \\
0 & M_{N}
\end{array}\right)\left(\begin{array}{c}
l_{R} \\
\psi_{R}
\end{array}\right)
$$

where $m_{\ell}=y_{\ell}<\phi^{0}>$ with $y_{\ell}$ is leptonic Yukawa coupling and $v=\sqrt{2}<\phi^{0}>=246 \mathrm{GeV}$. On the other hand the symmetric mass matrix for the neutral states is given by

$$
\begin{aligned}
\mathcal{L} \ni & -\left(\overline{\nu_{L}} \overline{\Sigma^{0 c}}\right)\left(\begin{array}{cc}
0 & Y_{N}^{\dagger} v / 2 \sqrt{2} \\
Y_{N}^{*} v / 2 \sqrt{2} & M_{N} / 2
\end{array}\right)\left(\begin{array}{c}
\nu_{L}^{c} \\
\Sigma^{0}
\end{array}\right) \\
& -\left(\overline{\nu_{L}^{c}} \overline{\Sigma^{0}}\right)\left(\begin{array}{cc}
0 & Y_{N}^{T} v / 2 \sqrt{2} \\
Y_{N} v / 2 \sqrt{2} & M_{N} / 2
\end{array}\right)\left(\begin{array}{c}
\nu_{L} \\
\Sigma^{0 c}
\end{array}\right) .
\end{aligned}
$$

The neutrino mass matrix in this case can be written as

$$
\mathcal{M}_{\nu}=\left(\begin{array}{cc}
0 & M_{D} \\
M_{D}^{\top} & M_{N}
\end{array}\right) \quad \text { where } \quad M_{D}=\frac{v}{\sqrt{2}} Y_{N}
$$

Thus the light neutrino mass can be written as

$$
m_{\nu}=-\frac{v^{2}}{2} Y_{N}^{T} \frac{1}{M_{N}} Y_{N}
$$

which mixes the left-handed neutrinos and the neutral fermionic triplet components (righthanded neutrinos). This leads to the full mass matrix for the neutral states as:

$$
\mathcal{M}_{\nu}=\left(\begin{array}{cc}
0 & M_{D} \\
M_{D}^{\top} & M_{N}
\end{array}\right)
$$

Diagonalizing the mass matrix we obtain one small neutrino eigenvalue in the limit of $\left\|M_{D}\right\| \ll\left\|M_{N}\right\|$ as:

$$
m_{\nu} \simeq-M_{D} M_{N}^{-1} M_{D}^{\top}
$$

where the triplet fermions take the mass values around $M_{N}$. From eq. (2.13), it is evident that to have the light neutrino mass $m_{\nu} \lesssim 0.1 \mathrm{eV}$, we need $Y_{N} \lesssim \mathcal{O}\left(10^{-6}\right)$ for $M_{N} \sim$ $\mathcal{O}(100) \mathrm{GeV}$. However, such choices of small couplings cannot affect the RG evolution of other couplings [54], a coupling motivated by inverse seesaw can be relevant here.

The collider signatures of heavy neutrinos and leptons rely upon larger Yukawa couplings. These are further restricted from electroweak precision data [110-114]. In the inverse seesaw frame work [115-118], we introduce another set of fermions which are $\mathrm{SU}(2)_{L}$ triplet, $\Sigma_{2 i}$ (with $i=1,2,3$ ) accompanying the $\Sigma_{1 i}$. The inverse seesaw Lagrangian is given by,

$$
\begin{aligned}
\mathcal{L}_{\text {ISS }}= & \operatorname{Tr}\left[\overline{\Sigma_{1 i}} \not D \Sigma_{1 i}\right]+\operatorname{Tr}\left[\overline{\Sigma_{2 i}} \not D \Sigma_{2 j}\right]-\frac{1}{2} \operatorname{Tr}\left[\overline{\Sigma_{2 i}} \mu_{\Sigma_{i j}} \Sigma_{2 j}^{c}+\overline{\Sigma_{2 i}^{c}} \mu_{\Sigma_{i j}}^{*} \Sigma_{2 j}\right] \\
& -\left(\widetilde{\Phi}_{1}^{\dagger} \overline{\Sigma_{1 i}} \sqrt{2} Y_{N_{i j}} L_{j}+\operatorname{Tr}\left[\bar{\Sigma}_{1 i} M_{N_{i j}} \Sigma_{2 j}\right]+\text { H.c. }\right)
\end{aligned}
$$


where $M_{D}$ is a $3 \times 3$ Dirac mass mixing matrix, $M_{N}$ is the mixing mass term between the two triplets and $\mu_{\Sigma}$ is the small lepton number violating mass term for the $\Sigma_{2}$-fields. In the basis of $\left\{\nu_{L}^{c}, \Sigma_{1}^{0}, \Sigma_{2}^{0}\right\}$, the full $9 \times 9$ neutral components mass matrix can be written as

$$
\mathcal{M}_{\nu}=\left(\begin{array}{ccc}
0 & M_{D} & 0 \\
M_{D}^{\top} & 0 & M_{N} \\
0 & M_{N}^{\top} & \mu_{\Sigma}
\end{array}\right)
$$

one obtains the three light neutrinos diagonalizing the mass matrix eq. (2.15) as described below

$$
m_{\nu} \simeq M_{D} M_{N}^{-1} \mu_{\Sigma}\left(M_{N}^{\top}\right)^{-1} M_{D}^{\top}
$$

other neutrino masses are heavy and they are given by $M_{N} \pm \mu_{\Sigma} / 2$. Here the small $\mu_{\Sigma}$ share the load of seesaw making $Y_{N} \sim \mathcal{O}(0.1)$. Such large Yukawa couplings give significant negative contributions in the stability of EW vacuum [119].

\section{Perturbativity}

To illustrate the theoretical bounds from perturbativity behaviour of the dimensionless couplings, we impose the following conditions on the dimensionless couplings as perturbative limit at a given scale $\mu$,

$$
\left|\lambda_{i}\right| \leq 4 \pi, \quad\left|g_{j}\right| \leq 4 \pi, \quad\left|Y_{k}\right| \leq \sqrt{4 \pi},
$$

where $g_{j}$ with $j=1,2$ are the EW gauge couplings, ${ }^{1} \lambda_{i}$ with $i=1,2,3,4,5$ are the quartic couplings corresponding to the scalars and $Y_{k}$ with $k=u, d, \ell$ are the Yukawa couplings for the quarks and leptons. The extension of SM with a $\mathrm{SU}(2)_{L}$ inert doublet as well as by $\mathrm{SU}(2)_{L}$ triplet fermions can change the running $g_{2}(\mu)$, which in turn affects the progression of other couplings namely the $\lambda_{i}$ relevant for the vacuum stability and perturbativity. Below we discuss that how $g_{2}(\mu)$ gets affected via the extra scalar and fermions of this model.

\subsection{Running of gauge couplings}

Eqs. (3.2)-(3.4) and figure 1(c) describe the evolution of the SM gauge couplings at a given scale $\mu$ (not explicitly mentioned in those eqs.) at two-loop level. Both $g_{2}$ and $g_{3}$ decrease with the increase in the running scale $\mu$ and remain perturbative in the high scale limit. However, such behaviour can change substantially with the inclusion of other $\mathrm{SU}(2)_{L}$ fields such as ID and Type-III Seesaw fermions, which are in the triplet of $\mathrm{SU}(2)_{L}$ representation.

$$
\begin{aligned}
\beta_{g_{1}}= & \frac{1}{16 \pi^{2}}\left[\frac{21}{5} g_{1}^{3}\right]+\frac{1}{\left(16 \pi^{2}\right)^{2}}\left[\frac { 1 } { 5 0 } g _ { 1 } ^ { 3 } \left(180 g_{2}^{2}+208 g_{1}^{2}-25 \operatorname{Tr}\left(Y_{d} Y_{d}^{\dagger}\right)\right.\right. \\
& \left.\left.+440 g_{3}^{2}-45 \operatorname{Tr}\left(Y_{N} Y_{N}^{\dagger}\right)-75 \operatorname{Tr}\left(Y_{e} Y_{e}^{\dagger}\right)-85 \operatorname{Tr}\left(Y_{u} Y_{u}^{\dagger}\right)\right)\right],
\end{aligned}
$$

\footnotetext{
${ }^{1}$ The running of $g_{3}$ remains the as in the $\mathrm{SM}$, as the new fields do not have $\mathrm{SU}(3)$ charges.
} 


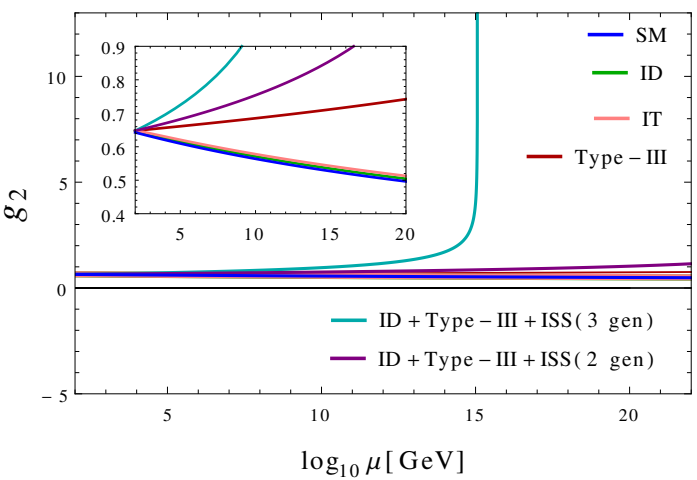

(a)

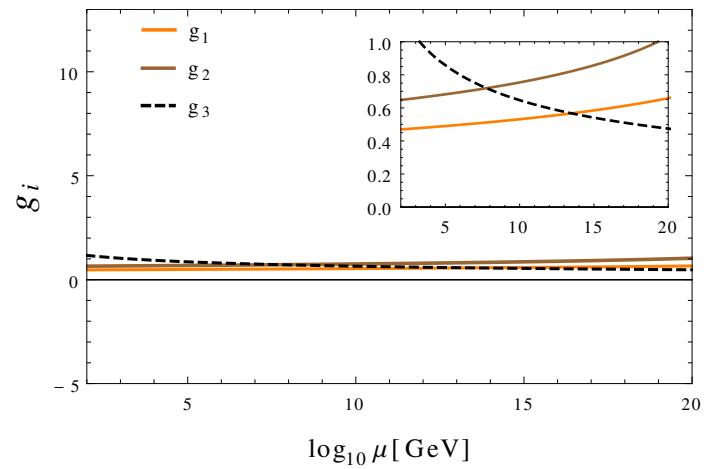

(b)

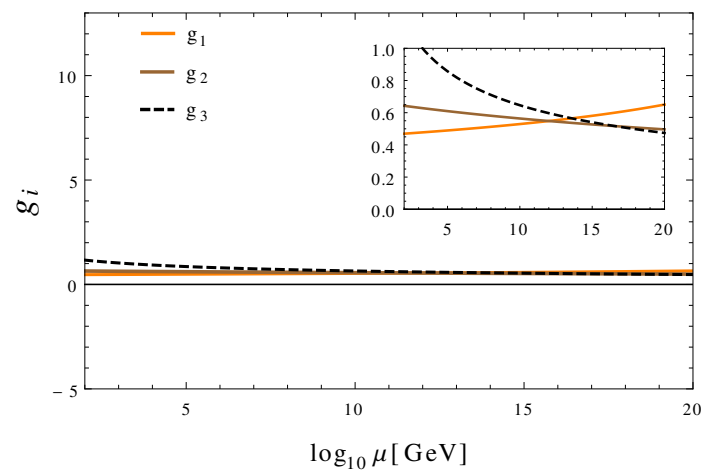

(c)

Figure 1. (a) Running of $g_{2}$ with the scale $\mu$ for the SM in blue, SM + ID in green, SM + ITM in pink, SM + three generations of Type-III fermions in red, SM+ID + three generations of Type-III fermions in sky-blue and SM+ ID+ two generations of Type-III fermions in purple in two-loop. (b) Running of all three gauge couplings $g_{i}$ at two-loop for the SM+ ID+ two generations of Type-III fermions. (c) Running of all three gauge couplings $g_{i}$ at two-loop for the SM.

$$
\begin{aligned}
\beta_{g_{2}}= & \frac{1}{16 \pi^{2}}\left[-\frac{19}{6} g_{2}^{3}\right]+\frac{1}{\left(16 \pi^{2}\right)^{2}}\left[\frac { 1 } { 3 0 } g _ { 2 } ^ { 3 } \left(-15 \operatorname{Tr}\left(Y_{e} Y_{e}^{\dagger}\right)+175 g_{2}^{2}+27 g_{1}^{2}\right.\right. \\
& \left.\left.+360 g_{3}^{2}-45 \operatorname{Tr}\left(Y_{d} Y_{d}^{\dagger}\right)-45 \operatorname{Tr}\left(Y_{u} Y_{u}^{\dagger}\right)\right)\right], \\
\beta_{g_{3}}= & \frac{1}{16 \pi^{2}}\left[-7 g_{3}^{3}\right]+\frac{1}{\left(16 \pi^{2}\right)^{2}}\left[-\frac{1}{10} g_{3}^{3}\left(-11 g_{1}^{2}+20 \operatorname{Tr}\left(Y_{d} Y_{d}^{\dagger}\right)\right.\right. \\
& \left.\left.+20 \operatorname{Tr}\left(Y_{u} Y_{u}^{\dagger}\right)+260 g_{3}^{2}-45 g_{2}^{2}\right)\right] .
\end{aligned}
$$

In eqs. (3.5)-(3.9), we show the modified evolution of the gauge couplings in the presence of the ID and triplet fermions. Eq. (3.5) shows that an inclusion of inert $\mathrm{SU}(2)_{L}$ doublet makes $\beta_{g_{2}}$ less negative at one-loop and so as at two-loop. The behaviours can be verified from the figure 1(a), where the blue curve implies SM and green line represents $\mathrm{SM}$ with ID. For the comparison we also show that an addition of a $Y=0 \mathrm{SU}(2)_{L}$ triplet scalar also reduces the negative impact in $\beta_{g_{2}}$, as can be read from eq. (3.6), which is 
also evident from figure 1(a) pink curve. However, from eq. (3.7) we can see that even at one-loop $\beta_{g_{2}}$ has become positive with the factor changed to $\frac{5 g_{2}^{3}}{6}$ if we extend the SM with three generations of Type-III fermions. This behaviour continues even at two-loop. The running of $g_{2}$ in this case has been depicted by red curve of figure 1(a). Unlike the $\mathrm{SM}$, or ID scenario, the coupling $g_{2}$ in this case increases with increasing $\mu$. In addition to the three generations of Type-III fermions if we add a $\mathrm{SU}(2)_{L}$ inert doublet which gives the much needed DM candidate, the factor at one-loop is enhanced to $5 g_{2}^{3}$ with further enhancement at two-loop and also visible by the sky-blue curve in figure 1(a). Certainly we can see that $g_{2}$ coupling remians perturbative till Planck scale for all the scenarios except ID + Type-III + ISS scenario with three generations of fermionic triplet. It is evident that with three generations of fermionic triplet $g_{2}$ coupling loses perturbativity around $10^{15} \mathrm{GeV}$. However, restricting it only to two generations of triplet fermions, and one ID, this perturbativity scale pushes till the Planck scale. This prompt us to choose only two generations of Type-III fermions along with ID, rather than the three generations. The running of the gauge coupling $g_{2}$ for the two generation scenario is evident from the purple curve of figure 1(a), and also from eq. (3.9). Figure 1(b) represents running of all the three gauge couplings for the two generation scenario. Therefore, from EW scale point of view, SM extension with an ID and two generations of Type-III fermions with ISS mechanism are more motivated, as this accommodates perturbativity of the gauge couplings till Planck scale, as well as, can explain two small light neutrino masses, and provide a dark matter candidate from the ID. However, one lives with the different asymptotic behaviour of $g_{2}$ as compared to SM.

$$
\begin{aligned}
\beta_{g_{2}^{\mathrm{IDM}}}= & \frac{1}{16 \pi^{2}}\left[-3 g_{2}^{3}\right]+\frac{1}{\left(16 \pi^{2}\right)^{2}}\left[\frac { 1 } { 1 0 } g _ { 2 } ^ { 3 } \left(120 g_{3}^{2}+12 g_{1}^{2}-15 \operatorname{Tr}\left(Y_{d} Y_{d}^{\dagger}\right)\right.\right. \\
& \left.\left.-15 \operatorname{Tr}\left(Y_{u} Y_{u}^{\dagger}\right)-5 \operatorname{Tr}\left(Y_{e} Y_{e}^{\dagger}\right)+80 g_{2}^{2}\right)\right] . \\
\beta_{g_{2}^{\mathrm{ITM}}=} & \frac{1}{16 \pi^{2}}\left[-\frac{17}{6} g_{2}^{3}\right]+\frac{1}{\left(16 \pi^{2}\right)^{2}}\left[\frac { 1 } { 3 0 } g _ { 2 } ^ { 3 } \left(-15 \operatorname{Tr}\left(Y_{e} Y_{e}^{\dagger}\right)+27 g_{1}^{2}+360 g_{3}^{2}\right.\right. \\
& \left.\left.+455 g_{2}^{2}-45 \operatorname{Tr}\left(Y_{d} Y_{d}^{\dagger}\right)-45 \operatorname{Tr}\left(Y_{u} Y_{u}^{\dagger}\right)\right)\right] . \\
\beta_{g_{2}, 3 \text { gen }}^{\text {yypeIII }}= & \frac{1}{16 \pi^{2}}\left[\frac{5}{6} g_{2}^{3}\right]+\frac{1}{\left(16 \pi^{2}\right)^{2}}\left[\frac { 1 } { 6 0 } g _ { 2 } ^ { 3 } \left(-165 \operatorname{Tr}\left(Y_{e} Y_{e}^{\dagger}\right)-30 \operatorname{Tr}\left(Y_{e} Y_{e}^{\dagger}\right)\right.\right. \\
& \left.\left.+4190 g_{2}^{2}+54 g_{1}^{2}+720 g_{3}^{2}-90 \operatorname{Tr}\left(Y_{d} Y_{d}^{\dagger}\right)-90 \operatorname{Tr}\left(Y_{u} Y_{u}^{\dagger}\right)\right)\right] \\
\beta_{g_{2}, 3 \text { gen }}= & \frac{1}{16 \pi^{2}}\left[5 g_{2}^{3}\right]+\frac{1}{\left(16 \pi^{2}\right)^{2}}\left[\frac { 1 } { 1 0 } g _ { 2 } ^ { 3 } \left(120 g_{3}^{2}+12 g_{1}^{2}+1360 g_{2}^{2}-15 \operatorname{Tr}\left(Y_{d} Y_{d}^{\dagger}\right)\right.\right. \\
& \left.\left.-15 \operatorname{Tr}\left(Y_{u} Y_{u}^{\dagger}\right)-55 \operatorname{Tr}\left(Y_{N} Y_{N}^{\dagger}\right)-5 \operatorname{Tr}\left(Y_{e} Y_{e}^{\dagger}\right)\right)\right] \\
& \frac{1}{16 \pi^{2}}\left[\frac{7}{3} g_{2}^{3}\right]+\frac{1}{\left(16 \pi^{2}\right)^{2}}\left[\frac { 1 } { 3 0 } g _ { 2 } ^ { 3 } \left(-15 \operatorname{Tr}\left(Y_{e} Y_{e}^{\dagger}\right)-165 \operatorname{Tr}\left(Y_{N} Y_{N}^{\dagger}\right)\right.\right. \\
& \left.\left.+2800 g_{2}^{2}+360 g_{3}^{2}+36 g_{1}^{2}-45 \operatorname{Tr}\left(Y_{d} Y_{d}^{\dagger}\right)-45 \operatorname{Tr}\left(Y_{u} Y_{u}^{\dagger}\right)\right)\right]
\end{aligned}
$$




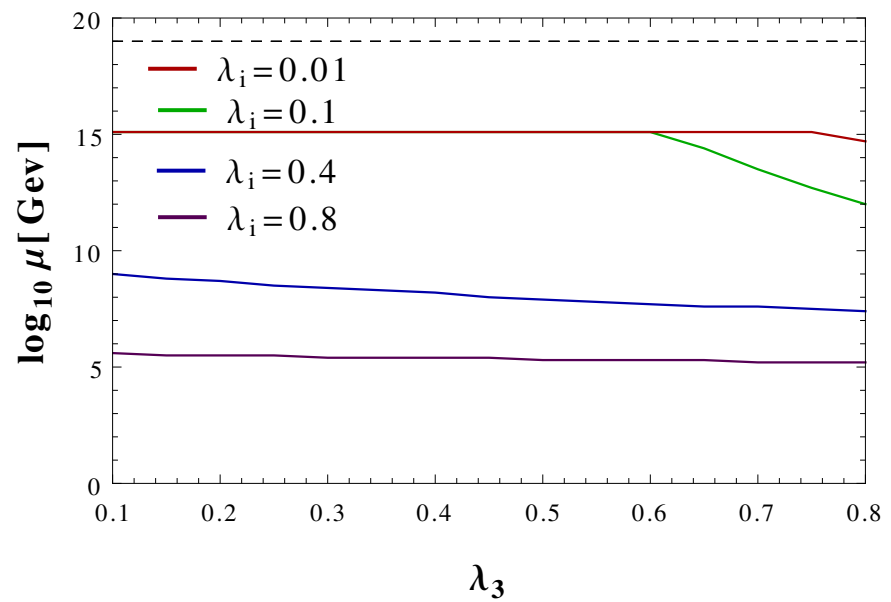

Figure 2. Bounds on Perturbative scale of the scalar quartic couplings verses $\lambda_{3}$ at two-loop for Yukawa coupling $Y_{N}=0.02$, considering three generations of fermionic triplet. Different initial values are designated as red, green, blue and purple curves for $\lambda_{i}=0.01,0.1,0.4,0.8$ respectively at the EW scale.

The perturbative nature of the scalar quartic couplings are also modified by the inclusion of ID and triplet fermions. Below, we first focus on the analysis of the perturbative behaviour of the scalar quartic couplings with respect to $\lambda_{3}$ in the scenario with SM associated with an ID, and three generations of fermionic triplet. The perturbative limit is calculated if at least one of the coupling crosses the perturbativity or hits Landau pole. Figure 2 describes the perturbative behaviour of $\lambda_{3}$ with the scale $\mu$ for $Y_{N}=0.02$, where the other quartic couplings $\lambda_{(i=2,4,5)}$ are kept at different values at the electroweak scale. For the coupling $\lambda_{1}$, we choose $\lambda_{1}=0.1264$, which gives SM like Higgs boson mass around $125.5 \mathrm{GeV}$. Here $\lambda_{i}, i=2,4,5$ at the EW scale are chosen in very weak ( $\lambda_{i}=0.01$ in red), weak $\left(\lambda_{i}=0.10\right.$ in green $)$, moderate $\left(\lambda_{i}=0.40\right.$ in blue $)$ and strong $\left(\lambda_{i}=0.80\right.$ in purple $)$ coupling limits respectively.

In ID+Type-III+ ISS scenario we have three generations of fermionic triplet $\Sigma_{1 i}$ which generates the Dirac term and three generations of additional fermionic triplet $\Sigma_{2 j}$ instrumental for inverse seesaw mechanism. Having $\mathrm{SU}(2)_{\mathrm{L}}$ charge they contribute to the beta functions of the $\mathrm{SU}(2)_{\mathrm{L}}$ gauge coupling, i.e. $\beta_{g_{2}}$ positively; which is somewhat different than normal Type-I + ISS case [54]. Additionally $\mathrm{Y}_{\mathrm{N}}$ also contributes positively to the beta functions of $\lambda_{3,4,5}$ at one-loop and negative effects only comes at two-loop. Both $\mathrm{Y}_{\mathrm{N}}$ and $\beta_{g_{2}}$ push the $\lambda_{3,4,5}$ towards non-perturbative limit. The Higgs quartic couplings $\lambda_{1}$ get negative corrections from the Yukawa coupling $Y_{N}$, pushing the Higgs potential toward instability at one-loop and two-loop. The detailed two-loop beta functions are given in appendix A, where only two generation effects are shown. However, in figure 2 we have considered all three generations of $\mathrm{SU}(2)_{\mathrm{L}}$ triplet fermions along with ID which makes the theory more stable, but simultaneously becomes non-perturbative below Planck scale for all corresponding values of $\lambda_{i}$ and $\mathrm{Y}_{\mathrm{N}}$. Thus for the Planck scale perturbativity we should restrict ourselves to two generations of fermionic triplets. For $\lambda_{i}=0.01,0.10$ which is less 
than $\lambda_{1}=0.1264, \lambda_{1}$ hits Landau pole before going into instability around $10^{15} \mathrm{GeV}$ (red and green lines). This happens due to positive effect of $g_{2}$, which is different than the Type-I case [54]. The bending happens due to further positive effects of $\lambda_{1} \operatorname{Tr}\left(\mathrm{Y}_{\mathrm{N}}^{\dagger} \mathrm{Y}_{\mathrm{N}}\right)$ and other quadratic terms involving $\lambda_{i} \mathrm{~s}$. Such bending effects grows from $\lambda_{i}=0.01$ (brown line) to $\lambda_{i}=0.10$ (green line). For $\lambda_{\mathrm{i}} \geq 0.2$ the perturbative limits come at much smaller case as other $\lambda_{i}$ s hit the Landau pole before $\lambda_{1}$.

In figure 3 , we present the perturbative behaviour of the Higgs quartic couplings for SM extension with ID + Type-III + ISS with two generations of fermionic triplets. The perturbativity behaviour of the scalar quartic couplings $\lambda_{3,4,5}$ are studied in figures $3(\mathrm{a})-3(\mathrm{f})$ respectively for two different choices of the coupling $Y_{N}$, i.e. $Y_{N}=0.01,0.40$. The other quartic couplings $\lambda_{i}$ are chosen to be $0.01,0.1,0.4$ and 0.8 which are shown by the red, green, blue and purple lines, respectively. Higgs quartic coupling $\lambda_{3}$ is perturbative till Planck scale for $\lambda_{3} \lesssim 0.56,0.37$ for $\lambda_{i}(\mathrm{EW})=0.01,0.10$ respectively, as shown in figure 3(a). For a larger coupling $\lambda_{i}(\mathrm{EW})=0.40,0.80$ theory becomes non-perturbative at much lower scale $\sim 10^{8.9}, 10^{5.7} \mathrm{GeV}$ for almost all initial values of $\lambda_{3}$, and for a coupling $Y_{N}=0.01$. Figure 3(b) shows the similar behaviour of $\lambda_{3}$ for a larger $Y_{N}$, where we choose $Y_{N}=0.40$ and the other quartic couplings $\lambda_{i}(\mathrm{EW})=0.01,0.10$. As is evident from figure $3(\mathrm{~b})$, the Higgs quartic coupling $\lambda_{3}$ is perturbative till Planck scale for $\lambda_{3} \lesssim 0.47,0.26$ for the above choices of $\lambda_{i}(\mathrm{EW})$. For $\lambda_{i}(\mathrm{EW})=0.40,0.80$, theory again becomes non-perturbative at much lower scale $\sim 10^{8.4}, 10^{5.4} \mathrm{GeV}$ for almost all initial values of $\lambda_{3}$. Similarly, the perturbative bounds on Higgs quartic coupling $\lambda_{4}$ are shown in figures $3(\mathrm{c})-3(\mathrm{~d})$. The results are very similar to the case of $\lambda_{3}$. Here for the choice of $\lambda_{i}(\mathrm{EW})=0.01,0.10$ the perturbative limits remain valid till the Planck scale for $\lambda_{4} \lesssim 0.64,0.36$ and for $Y_{N}=0.01$. For a larger $Y_{N}=0.40$, the corresponding perturbative limit turns out to be $\lambda_{4} \lesssim 0.54,0.24$. For higher values of $\lambda_{i}(\mathrm{EW})$, theory becomes non-perturbative at much lower scale, i.e. $\sim 10^{9.2}, 10^{6.1} \mathrm{GeV}$ for $Y_{N}=0.01$ and $\sim 10^{8.8}, 10^{5.9} \mathrm{GeV}$ for almost all initial values of $\lambda_{4}$ and for the choice of Yukawa couplings $Y_{N}=0.01$ and $Y_{N}=0.40$, respectively. As depicted in figures 3(e)-3(f), Higgs quartic coupling $\lambda_{5}$ is perturbative till Planck scale for $\lambda_{5} \lesssim 0.29,0.22$ for $Y_{N}=0.01$ and $\lambda_{5} \lesssim 0.24,0.16$ for $Y_{N}=0.40$ for the choice of $\lambda_{i}(\mathrm{EW})=0.01,0.10$ respectively. For higher values of $\lambda_{i}(\mathrm{EW})$, theory becomes nonperturbative at much lower scale $\sim 10^{10.1}, 10^{6.2} \mathrm{GeV}$ for $Y_{N}=0.01$ and $\sim 10^{9.8}, 10^{6.1} \mathrm{GeV}$ for $Y_{N}=0.4$ respectively for almost all initial values of $\lambda_{5}$. The perturbative scale decreases for larger choices of $Y_{N}$ as $\lambda_{3,4,5}$ increases with $Y_{N}$ and even faster than Type-I case [54] with the stringent constraint comes from the perturbativity bound of $\lambda_{5}$. Here the theory becomes non-perturbative before Planck scale for $\lambda_{i}(\mathrm{EW})=0.40,0.80$ respectively. For $\lambda_{i}(\mathrm{EW})=0.01,0.10$ the Planck scale validity can be achieved for $\lambda_{5} \leq 0.30,0.22$ and $\lambda_{5} \leq 0.25,0.17$ for the choices of $Y_{N}=0.01,0.40$ respectively.

Figure 4 depicts the bounds on perturbative scale $\left(\log _{10} \mu\right)$ with respect to the Yukawa coupling $Y_{N}$ for different choices of initial values of $\lambda_{(i=2,3,4,5)}$. For $Y_{N}$ values up to 0.1, the effect of triplet fermions on the perturbativity of $\lambda_{i}$ is hardly noticeable as shown in figure 4. The exact value of $Y_{N}$ where it starts affecting the perturbativity depends on the initial value of $\lambda_{i}$ at EW scale. However, due to enhanced $g_{2}$ in the case of Type-III, $\lambda_{i}$ attains perturbation limit before than Type-I case and the effect is more prominent 


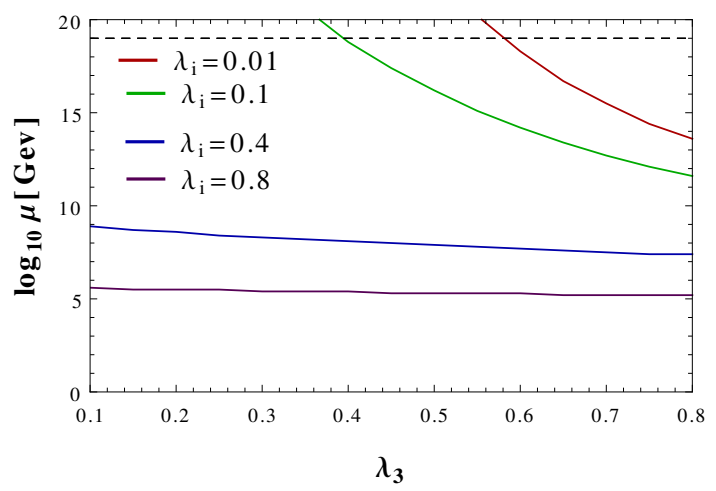

(a) $Y_{N}=0.01$

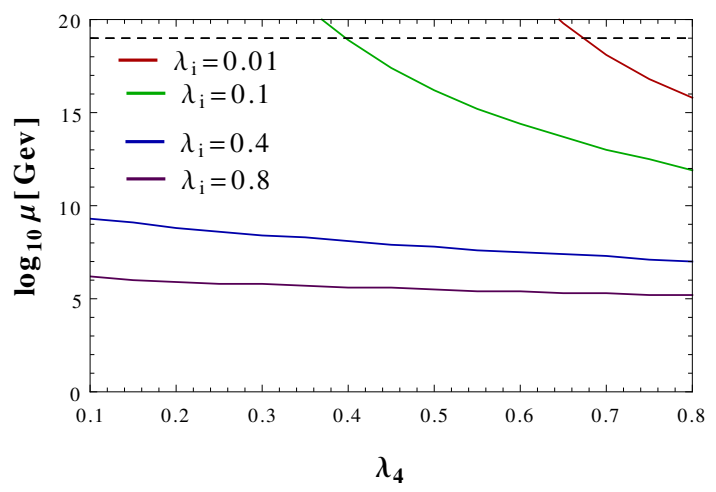

(c) $Y_{N}=0.01$

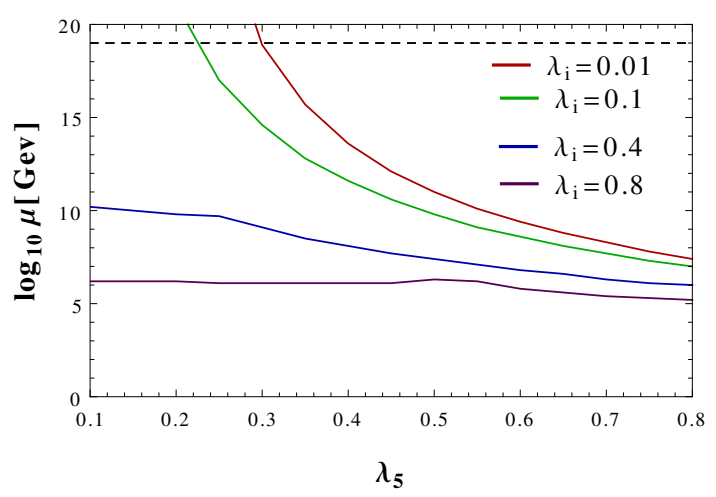

(e) $Y_{N}=0.01$

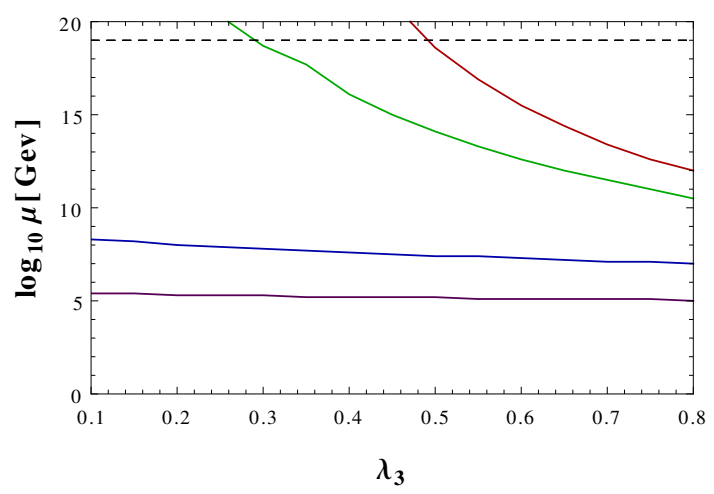

(b) $Y_{N}=0.4$

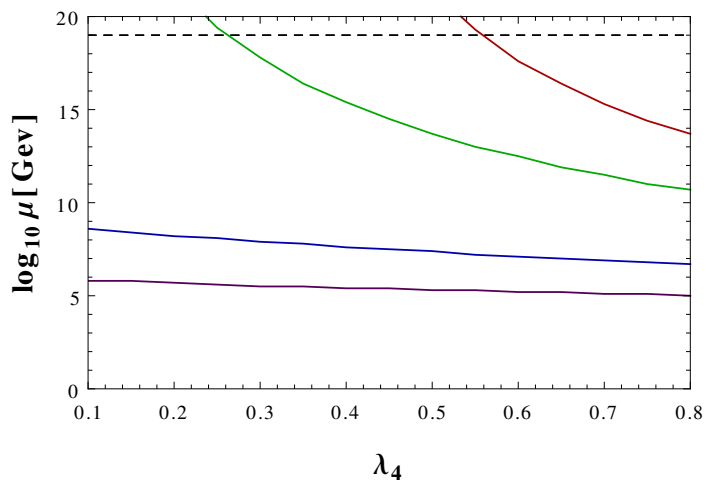

(d) $Y_{N}=0.4$

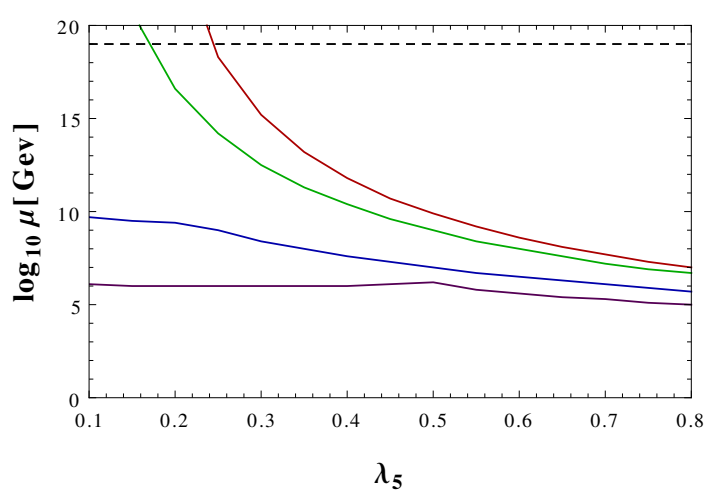

(f) $Y_{N}=0.4$

Figure 3. Perturbative limits of $\lambda_{3}, \lambda_{4}$ and $\lambda_{5}$ for various $\lambda_{i} \mathrm{~s}$ and $Y_{N}=0.01,0.40$ at EW scale. The colour codes are presented as: very weak $\left(\lambda_{i}=0.01\right.$ in red $)$, weak $\left(\lambda_{i}=0.10\right.$ in green $)$, moderate $\left(\lambda_{i}=0.40\right.$ in blue) and strong $\left(\lambda_{i}=0.80\right.$ in purple) couplings respectively with two generations of fermionic triplet in the SM plus ID plus Type-III ISS scenario. 


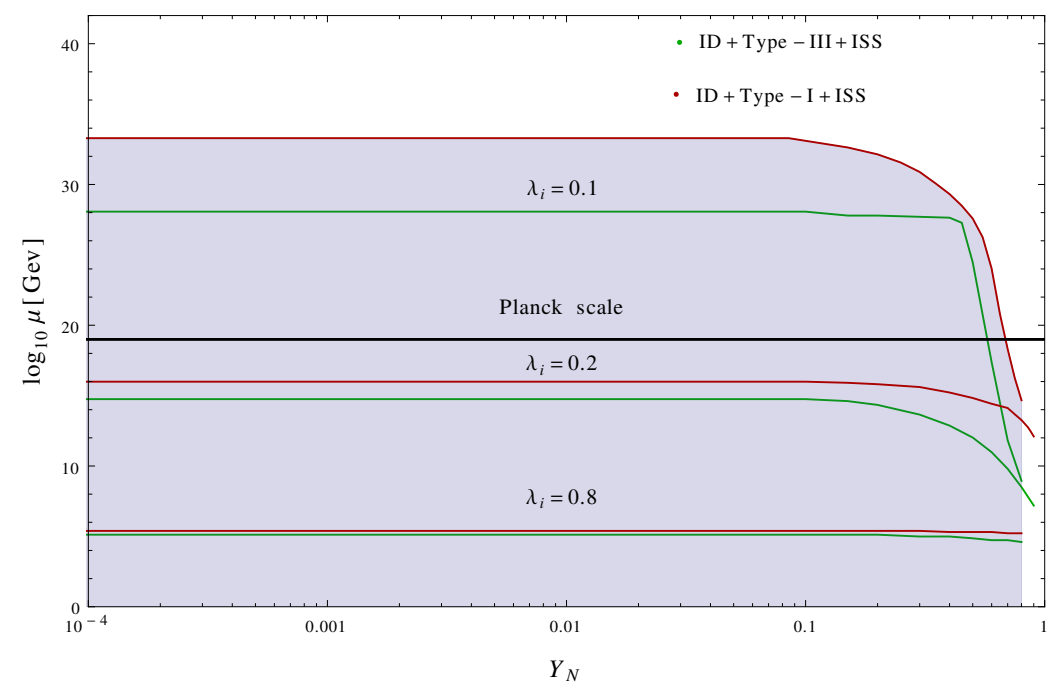

Figure 4. Bounds from perturbativity scale on $Y_{N}$ as a function of $\mu$ for different values of $\lambda_{i}$ with $y_{t}=0.9369, M_{N}=100 \mathrm{GeV}$. The red curve and the green curve corresponds to the ID plus TypeI ISS scenario and ID plus Type-III ISS scenario respectively with two generations of fermionic triplets.

for lower values of $\lambda_{i}(\mathrm{EW})$. For $\lambda_{i}(\mathrm{EW})=0.1$ in ID+Type-III+ISS, the $\lambda_{1}$ hits Landau pole before other $\lambda_{\mathrm{s}}$ as $\lambda_{1}(\mathrm{EW})=0.1264$ and gets large positive contribution form the enhanced $g_{2}$ in this case. Effects of other $\lambda_{i}$ s are negligible in this case until a particular value of $Y_{N}$. Large $g_{2}$ effect brings the perturbative scale to $10^{28.07} \mathrm{GeV}$ with prolonged effect till $Y_{N} \lesssim 0.23$ after which $\lambda_{1} \operatorname{Tr}\left(\mathrm{Y}_{\mathrm{N}}^{\dagger} \mathrm{Y}_{\mathrm{N}}\right)$ effect takes over bringing perturbative scale further down as can be seen from the green line with the bending effect. Compared to that in ID+Type-I+ISS case, for $\lambda_{i}=0.1$, the perturbativity scale is $\sim 10^{33.29} \mathrm{GeV}$ and the effect starts showing up for $Y_{N}>0.15$ in ID+Type-I+ISS as can be seen in red line. Here perturbative limits are obtained via the Landau poles of other $\lambda_{i}$ as $g_{2}$ decreases with the scale in this case and large $Y_{N}$ pushes $\lambda_{1}$ towards negative values of instability [54]. However, other $\lambda_{i}$ gets positive contributions $\lambda_{\mathrm{i}} \operatorname{Tr}\left(\mathrm{Y}_{\mathrm{N}}^{\dagger} \mathrm{Y}_{\mathrm{N}}\right)$ towards their Landau pole. Like the Type-III case here also being very small $\lambda_{i}$ effects are negligible.

For higher values of $\lambda_{i}(\mathrm{EW})$, the perturbativity limits are obtained when other $\lambda \mathrm{s}$ hit Landau pole before $\lambda_{1}$ and mostly controlled by $\lambda_{i}$ (see appendix A) and $\lambda_{1}$ runs towards instability by large $Y_{N}$ effect. This also results in lesser splitting in the perturbative scale between Type-III and Type-I until $\lambda_{\mathrm{i}} \operatorname{Tr}\left(\mathrm{Y}^{\dagger} \mathrm{Y}_{\mathrm{N}}\right)$ effects creep in with larger factor for TypeIII as compared to Type-I. For $\lambda_{i}=0.2$, the perturbativity limit comes around the GUT scale $10^{16-15} \mathrm{GeV}$ and the fermion effect starts for $Y_{N} \gtrsim 0.3$ in both scenarios but the effect of $Y_{N}$ is much stronger in ID+Type-III+ISS.

For further higher values of $\lambda_{i}=0.8$, the perturbativity scales are almost the same $\sim$ $10^{5} \mathrm{GeV}$ for both Type-I and Type-III cases mostly governed by the $\lambda_{i}$ effects. The effect of new fermion comes much later for $Y_{N}>0.60$. Higher values of $\lambda_{i}$ s can accommodate higher values of $Y_{N}$ for vacuum stability in $\lambda_{1}$ direction. On the other hand, the theory becomes 
non-perturbative at much lower scale. One can read the upper bounds on perturbativity from figure 4 for $\lambda_{i}$ and $Y_{N}$ values. We see that for the $\lambda_{i} \leq 0.15$ and $Y_{N} \leq 0.25, Y_{N} \leq 0.3$ the theory remains perturbative till the Planck scale for Type-III and Type-I respectively.

\section{Stability bound}

In this section we analyse the stability of Higgs potential via two different approaches. Firstly via calculating two-loop scalar quartic couplings and checking if the SM Higgs like quartic coupling $\lambda_{h}$ becomes negative at some higher scale. In this case $\lambda_{h}=\lambda_{1}$ at treelevel, but at one-loop and two-loop levels $\lambda_{h}$ gets contribution from SM fields as well as the ID and the Type-III fermions. The details are provided in subsection 4.1. For simplicity, we only present the expressions of the corresponding beta functions at one-loop in the next subsection. The expressions for the two-loop beta functions are given in the appendix A. Secondly we follow the effective potential approach as described in detail in section 4.3.

\subsection{RG evolution of the scalar quartic couplings}

To study the evolutions of dimensionless couplings we have implemented the ID + Type-III Seesaw + ISS scenario in SARAH 4.13.0 [120] for two generations $\mathrm{SU}(2)_{L}$ triplet fermions. The corresponding $\beta$-functions for various gauge, quartic and Yukawa couplings are calculated at one- and two-loop. The full two-loop $\beta$-functions can be found in appendix A, and used for our numerical analysis of vacuum stability which will be described here. We first look at the one-loop $\beta$-function of $\lambda_{h}$ to observe the effect of the Yukawa and other scalar quartic couplings. $\lambda_{h}=\lambda_{1}$ at tree-level and effects of other particles start entering at one-loop level. The $\beta$-function of the SM-like Higgs quartic coupling $\lambda_{h}$ in this model receives three different types of contributions: one from the SM gauge, Yukawa, quartic interactions, the second from the Type-III Seesaw Yukawa couplings, and the third from the inert scalar sector (ID):

$$
\beta_{\lambda_{h}}=\beta_{\lambda_{1}}=\beta_{\lambda_{1}}^{\mathrm{SM}}+\beta_{\lambda_{1}}^{\mathrm{Type}-\mathrm{III}+\mathrm{ISS}}+\beta_{\lambda_{1}}^{\mathrm{ID}},
$$

with

$$
\begin{aligned}
\beta_{\lambda_{1}}^{\mathrm{SM}}= & \frac{1}{16 \pi^{2}}\left[\frac{27}{200} g_{1}^{4}+\frac{9}{20} g_{1}^{2} g_{2}^{2}+\frac{9}{8} g_{2}^{4}-\frac{9}{5} g_{1}^{2} \lambda_{1}-9 g_{2}^{2} \lambda_{1}+24 \lambda_{1}^{2}\right. \\
& +12 \lambda_{1} \operatorname{Tr}\left(Y_{u} Y_{u}^{\dagger}\right)+12 \lambda_{1} \operatorname{Tr}\left(Y_{d} Y_{d}^{\dagger}\right)+4 \lambda_{1} \operatorname{Tr}\left(Y_{e} Y_{e}^{\dagger}\right) \\
& -6 \operatorname{Tr}\left(Y_{u} Y_{u}^{\dagger} Y_{u} Y_{u}^{\dagger}\right)-6 \operatorname{Tr}\left(Y_{d} Y_{d}^{\dagger} Y_{d} Y_{d}^{\dagger}\right) \\
& \left.-2 \operatorname{Tr}\left(Y_{e} Y_{e}^{\dagger} Y_{e} Y_{e}^{\dagger}\right)\right], \\
\beta_{\lambda_{1}}^{\text {Type-III+ISS }}= & \frac{1}{16 \pi^{2}}\left[12 \lambda_{1} \operatorname{Tr}\left(Y_{N} Y_{N}^{\dagger}\right)-10 \operatorname{Tr}\left(Y_{N} Y_{N}^{\dagger} Y_{N} Y_{N}^{\dagger}\right)\right], \\
\beta_{\lambda_{1}}^{\mathrm{ID}}= & \frac{1}{16 \pi^{2}}\left[2 \lambda_{3}^{2}+2 \lambda_{3} \lambda_{4}+\lambda_{4}^{2}+4 \lambda_{5}^{2}\right] .
\end{aligned}
$$

Here $Y_{u}, Y_{d}, Y_{e}$ represent the up, down and electron-type Yukawa couplings and other Yukawa couplings are neglected [9] and $g_{1}, g_{2}$ are the $\mathrm{U}(1)_{Y}, \mathrm{SU}(2)_{L}$ gauge couplings respectively their values at the EW scale [77] are: $\lambda_{1}=0.1264, g_{1}=0.3583, g_{2}=0.6478$, 


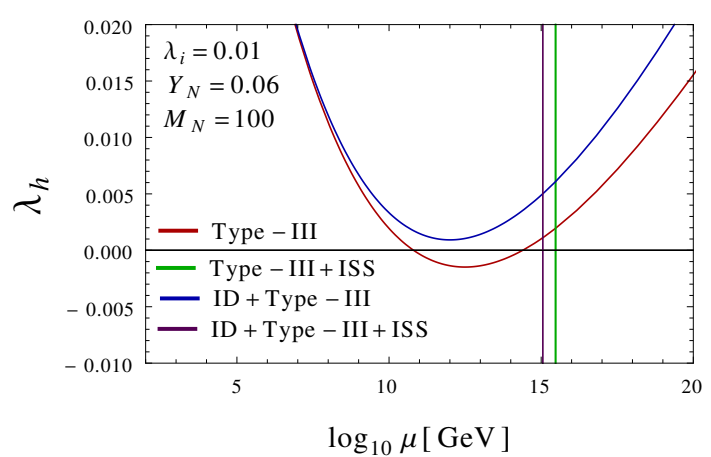

(a)

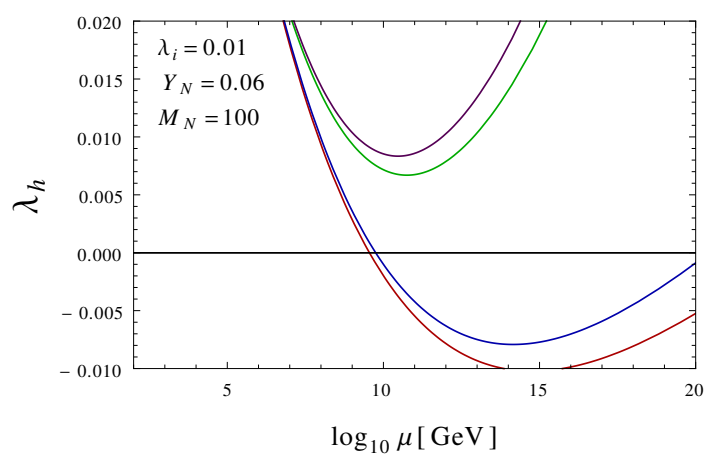

(b)

Figure 5. Two loop running of the SM like Higgs quartic coupling as a function of energy. Here the red, green, blue and the purple curve correspond to the Type-III Seesaw, Type-III Seesaw+ISS, ID+Type-III Seesaw and ID+Type-III Seesaw+ISS scenarios. The left and right plot represent three generations and two generations of fermionic triplets, respectively.

$y_{t}=0.9369$. In this analysis the ISS contribution to the RG evolution of $\lambda_{1}$ starts only at the scale above $M_{N}$.

In figures $5(\mathrm{a})-5(\mathrm{~b})$, we show the respective $\lambda_{h}$ running with the scale $\mu$. The plot in the left panel represents the three generation scenario, and the plot in the right panel represents two generations scenario. The two plots describe the behaviour of $\lambda_{h}$ at two-loop level for four different cases as before with three and two generations of triplet fermions, where $M_{N}=100 \mathrm{GeV}, \lambda_{1}=0.1264, \lambda_{i}=0.01$ (with $i=2,3,4,5$ ) at the EW scale. The RG evolution of $\lambda_{1} \equiv \lambda_{h}$ is described by the red curve as a function of the scale $\mu$ where $\lambda_{h}$ has contributions of $\beta_{\lambda_{1}}^{\mathrm{SM}}+\beta_{\lambda_{1}}^{\text {Type-III }}$ only. The green curve shows the evolution using $\beta_{\lambda_{1}}^{\mathrm{SM}}+\beta_{\lambda_{1}}^{\text {Type-III }}+\beta_{\lambda_{1}}^{\text {ISS }}$, blue curve describes the evolution using $\beta_{\lambda_{1}}^{\mathrm{SM}}+\beta_{\lambda_{1}}^{\text {Type-III }}+\beta_{\lambda_{1}}^{\text {ID }}$, and finally the purple curve shows the full evolution using $\beta_{\lambda_{1}} \equiv \beta_{\lambda_{1}}^{\mathrm{SM}}+\beta_{\lambda_{1}}^{\mathrm{Type}-\mathrm{III}}+\beta_{\lambda_{1}}^{\mathrm{ISS}}+\beta_{\lambda_{1}}^{\mathrm{ID}}$ [cf. eq. (4.1)]. The added effects of the new contributions to $\lambda_{1} \equiv \lambda_{h}$ at one-loop are given in eq. (4.1) and the detailed two-loop expressions are written in appendix A.

In figure 5(a), the three generations of fermionic triplet make the $g_{2}$ contribution too large (see eq. (3.8)) for both Type-III+ISS (green) and ID+ Type-III +ISS (purple), such that $\beta_{\lambda_{1}}$ hits the Landau pole at $\sim 10^{15.4} \mathrm{GeV}$ and $\sim 10^{15.1} \mathrm{GeV}$ respectively before hitting the instability scale (at which $\lambda_{h} \leq 0$ ). This makes the theory non-perturbative below Planck scale. Without ISS the $\beta_{g_{2}}$ is relatively smaller (see subsection 3.1) which restrains $\lambda_{h}$ from hitting the Landau pole. Thus for Type-III Seesaw scenario $\beta_{\lambda_{h}}$ becomes unstable $\sim 10^{10.7} \mathrm{GeV}$ but bounce back to stability (where $\lambda_{h} \geq 0$ ) at $\sim 10^{14} \mathrm{GeV}$. For ID+ Type-III scenario, $\beta_{\lambda_{h}}$ remains stable till Planck scale for $\lambda_{i}=0.01$ and $Y_{N}=0.06$ respectively.

Figure 5(b) describes the behaviour of two generations of fermionic triplet with reduced positive $g_{2}$ effect, which prohibits the Landau pole of $\lambda_{1}$. We can see the ID+Type-III+ ISS (purple curve) is more stable than Type-III+ISS (green curve). Again without ISS, the $g_{2}$ contribution is less so Type-III (red) and ID+Type-III (blue) have gone more negative; especially Type-III (red) is even more negative due to the lack of positive effects of ID scalars. 


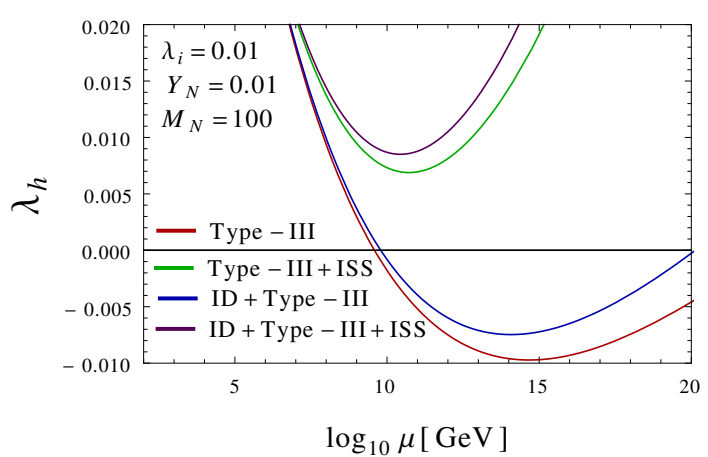

(a)

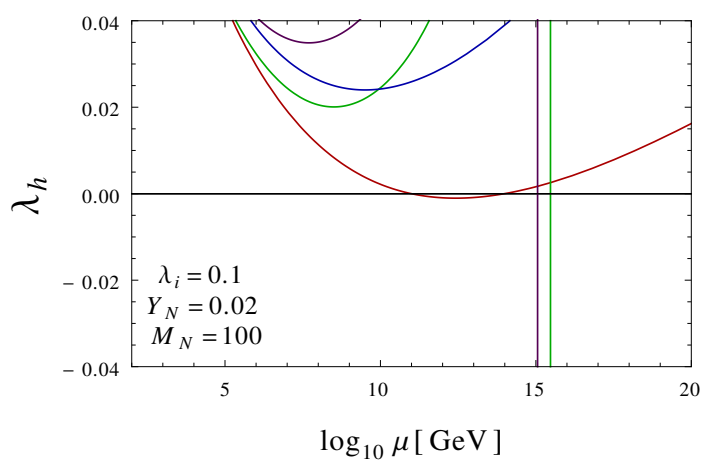

(c) 3 gen

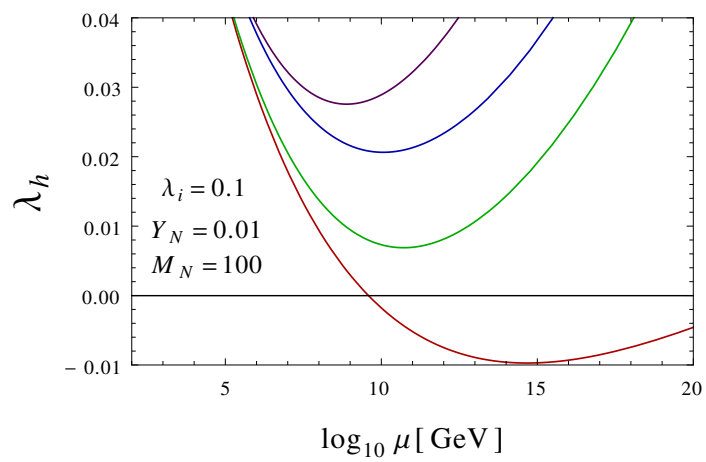

(e)

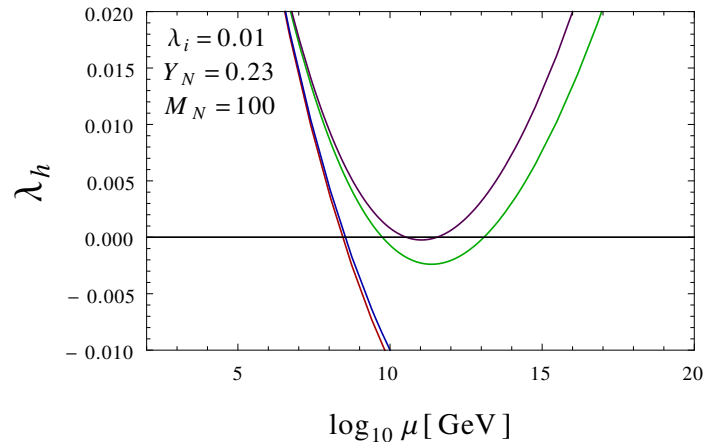

(b)

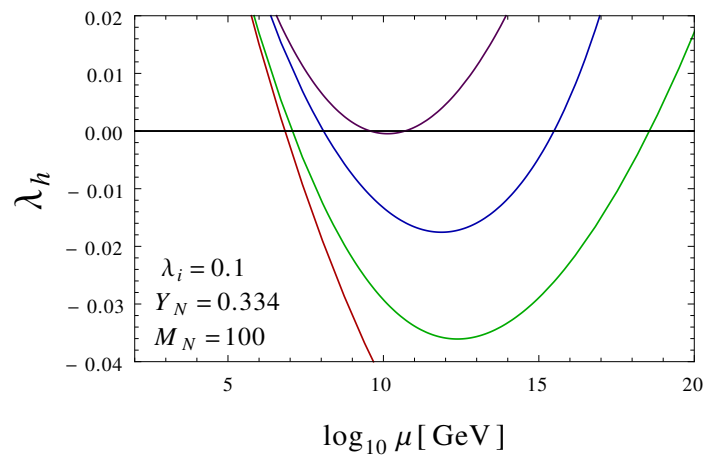

(d)

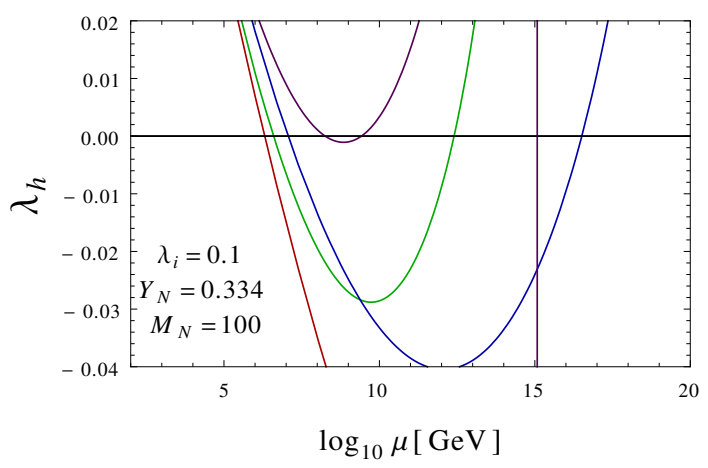

(f) 3 gen

Figure 6. Two-loop running of the SM-like Higgs quartic coupling $\lambda_{h}$ as a function of scale $(\mu)$. Here the red, green, blue and the purple curve corresponds to the Type-III, Type-III+ISS, ID+Type-III and ID+Type-III + ISS scenarios respectively for four different benchmark values of $Y_{N}$ and Higgs quartic couplings $\left(\lambda_{1=2,3,4,5}\right)$ with two generations of fermionic triplet. (c) and (f) are with three-generations of triplet fermions.

In figure 6 we describe the behaviour of two-loop running of the SM-like Higgs quartic coupling $\lambda_{h}$ as a function of scale $(\mu)$ for six benchmark points. We follow the same colour code as figure 5 and $\lambda_{1}(E W)=0.1264$ chosen for all the graphs. Figure 6(a) describes for the benchmark points of $\lambda_{i}=0.01, Y_{N}=0.01$ and $M_{N}=100 \mathrm{GeV}$ where $i=2,3,4,5$ 
with two generations of Type-III fermions. We can clearly see that the stability scales for ID+Type-III+ISS (purple curve) and Type-III+ISS (green) are enhanced to Planck scale. Type-III (red) and ID+ Type-III (blue) hit instability around $10^{9.5} \mathrm{GeV}$. Now if we increase $Y_{N}=0.23$ as shown in figure 6(b), we can see that red and blue curve hit instability earlier around $10^{8.5} \mathrm{GeV}$ due to larger negative effects of $Y_{N}$. Even green and purple curves also hit $\lambda_{h}=0$ due to this negative effect. For a comparative study with figure 6 (a) we plot the running of the $\lambda_{h}$ for three generations of Type-III fermions in figure 6(c). We see that the purple and the green curve hits Landau pole again due to larger positive $g_{2}$ contributions as explained earlier. Blue and red curve also move towards stability in this case. Figure 6(e) we restrict the fermion generation only to two and with the reduced positive effect the Landau poles are gone with overall shift towards the left.

Figure 6(d) shows the comparison with figure 6(b) with two Type-III fermion generation with $Y_{N}$ enhanced to 0.334 . We notice the overall negative effect that shifts all the curves towards the left reducing the instability scale with red curve hitting instability at first at $10^{7} \mathrm{GeV}$. If we increase the number of fermion generation to three in figure $6(\mathrm{f})$ the effect will be enhanced as red curve crosses zero at around $10^{6} \mathrm{GeV}$ before acquiring the Landau pole at $10^{15} \mathrm{GeV}$. The shapes of all the curves becomes more steeper as compared to figure $6(\mathrm{~d})$. Compared to figure $6(\mathrm{c})$ where $Y_{N}=0.02$, if we increase $Y_{N}=0.334$ in figure $6(\mathrm{f})$, we see that the negative effect creeps in and the Type-III + ISS i.e. green the green curve does not hit the Landau pole. However, in ID+Type-III +ISS case due to the positive contributions from $\lambda_{i}$ the purple curve hits the Landau pole at $10^{15} \mathrm{GeV}$.

\subsection{Variation perturbativity and stability with $Y_{N}$}

Figure 7 describes the variation of the stability scale with $Y_{N}$ and $\lambda_{i}$. We notice that for the smaller values of $\lambda_{i} \sim 0.1$ (green circles), the Planck scale stability can be achieved till $Y_{N} \leq 0.32$. In this case $\lambda_{1}$ strikes the perturbative bounds before other quartic couplings due to the strong $g_{2}$ effect and as $\lambda_{1}(E W)=0.1264$. The other quartic coupling effects are negligible in this region as explained earlier. After $Y_{N} \geq 0.32$ (green line) the negative contribution from the new fermions become very strong and push $\lambda_{h}$ towards negative values at around $10^{9} \mathrm{GeV}$.

For example as we enhance the $\lambda_{i}$ at EW scale the effect of quartic couplings in their beta functions increase as $\lambda_{\mathrm{i}} \operatorname{Tr}\left(\mathrm{Y}_{\mathrm{N}}^{\dagger} \mathrm{Y}_{\mathrm{N}}\right)$ along with the enhanced $g_{2}$ effect. These inflate $\beta_{\lambda_{1}}$ towards the higher scale stability compared to the SM but leads to non-perturbative limit for the other $\lambda_{i} \mathrm{~s}$ at lower scale. The point to be noted here is that for the choices of $\lambda_{i}>\lambda_{1}$ at the EW scale, other $\lambda_{i}(i=2,3,4,5)$ are most likely to hit the Landau pole before $\lambda_{1}$. For example as we enhance the $\lambda_{i}=0.2$ with $\lambda_{1}=0.1264$ at the EW scale, one of the $\lambda_{i}$ s afflicts the landau pole (shown by pink color diamond) around $10^{15}-10^{12} \mathrm{GeV}$, even before $\lambda_{1}$ enters into instability at $Y_{N}=0.47$ for $10^{6.24} \mathrm{GeV}$ (shown by pink line). The bending of the curves for higher $Y_{N}$ are due to the positive $\lambda_{\mathrm{i}} \operatorname{Tr}\left(\mathrm{Y}_{\mathrm{N}}^{\dagger} \mathrm{Y}_{\mathrm{N}}\right)$ effect for perturbativity and negative $\operatorname{Tr}\left(\mathrm{Y}_{\mathrm{N}}^{\dagger} \mathrm{Y}_{\mathrm{N}} \mathrm{Y}_{\mathrm{N}}^{\dagger} \mathrm{Y}_{\mathrm{N}}\right)$ effect for the instability respectively.

For $\lambda_{i}=0.3$, one of the $\lambda_{i}$ s hits the Landau pole at much lower scale around $10^{10} \mathrm{GeV}$ (cyan colour star) due to large quartic coupling contributions along with $\lambda_{\mathrm{i}} \operatorname{Tr}\left(\mathrm{Y}_{\mathrm{N}}^{\dagger} \mathrm{Y}_{\mathrm{N}}\right)$. In 


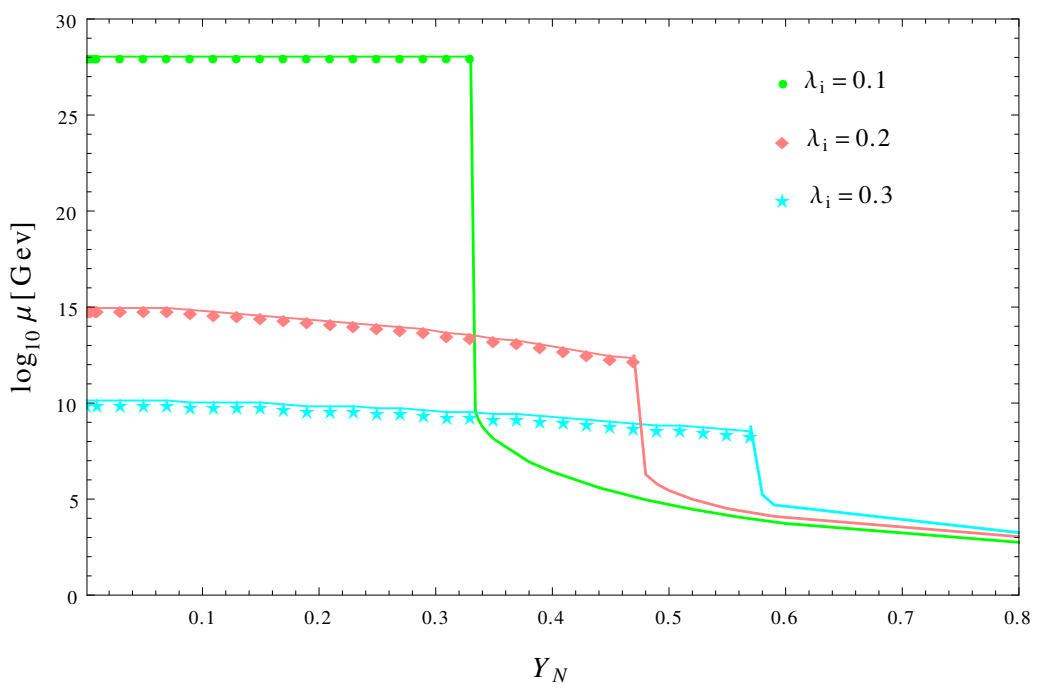

Figure 7. Effect of Yukawa coupling on the perturbativity and stability bound for different values of $\lambda_{i} . \lambda_{i}=0.10$ (green curve) ensures Planck scale perturbativity and stability for $Y_{N} \leq 0.32$. The pink curve corresponds to $\lambda_{i}=0.2$ which gives Landau pole for $Y_{N} \leq 0.47$ (shown by diamond). After $Y_{N}=0.47$, the negative fermionic contribution starts dominating which makes the $\lambda_{h} \leq 0$ for $10^{6.24} \mathrm{GeV}$ (shown by pink line) before hitting the Landau pole. For $\lambda_{i}=0.3$, the running of $\beta_{\lambda_{1}}$ hits the Landau pole till $Y_{N} \leq 0.57$ (described by cyan color star) and later the effect of fermions makes the $\lambda_{h} \leq 0$ for $10^{5.23} \mathrm{GeV}$ (described by cyan line).

this case the negative effect of $Y_{N}$ in $\lambda_{1}$ starts much later due to larger value of $\lambda_{i}$ and at $Y_{N} \geq 0.57$ the effect of fermions make the $\lambda_{h} \leq 0$ for $10^{5.23} \mathrm{GeV}$ (cyan line).

\subsection{Vacuum stability: RG-improved potential approach}

We study the stability of the EW vacuum via the Coleman and Weinberg [121] prescription of RG-improved effective potential at one-loop. The effective potential at one-loop has been calcualted for SM + Type-III-ISS + ID with two generations of fermions. The potential then analysed for the stability, metastability and instability by observing the behaviour of the effective Higgs quartic coupling. For this purpose we scan the parameter space of the model and then segregate them as above mentioned three regions.

Eq. (2.3) describes the tree-level Higgs potential for the model. The tree-level stability conditions of the potential are given by [108]

$$
\lambda_{1} \geq 0, \quad \lambda_{2} \geq 0, \quad \lambda_{3} \geq-\sqrt{\lambda_{1} \lambda_{2}}, \quad \lambda_{3}+\lambda_{4}-\left|\lambda_{5}\right| \geq-\sqrt{\lambda_{1} \lambda_{2}} .
$$

In SM $\lambda_{h}$ receives negative effects from the top quark loop which makes the potential unstable at around $10^{10-11} \mathrm{GeV}$. There is also a possibility of second minima in the $h$ direction due to quantum fluctuation. In this article we will see how different beyond SM fields can contribute to that possibility of second minima. However, such possibility can only occur at higher field values which justifies the choice of effective potential as 
given below:

$$
V_{\text {eff }}(h, \mu) \simeq \lambda_{\text {eff }}(h, \mu) \frac{h^{4}}{4}, \quad \text { with } h \gg v,
$$

where $\lambda_{\text {eff }}(h, \mu)$ is the effective Higgs quartic coupling and the calculation of which is described below. Then the stability of the vacuum then be guaranteed for the scale $\mu$ by assuring that $\lambda_{\text {eff }}(h, \mu) \geq 0$.

\subsection{Effective potential}

In our model the one-loop RG-improved effective potential can be written as

$$
V_{\text {eff }}=V_{0}+V_{1}^{\mathrm{SM}}+V_{1}^{\mathrm{ID}}+V_{1}^{\mathrm{ISS}+\mathrm{Type}-\mathrm{III}},
$$

where $V_{0}$ is the tree-level potential given by eq. (2.3), $V_{1}^{\mathrm{SM}}$ is the effective ColemanWeinberg potential in the SM that contains all the one-loop corrections involving the SM particles at zero temperature with vanishing momenta. The other two terms $V_{1}^{\mathrm{ID}}$ and $V_{1}^{\text {ISS+Type-III seesaw }}$ represent the corresponding one-loop effective potential terms from the inert scalar doublet, and fermionic triplet, respectively. $V_{1}$ describes one-loop Coleman and Weinberg effective potential which can be written as

$$
V_{1}(h, \mu)=\frac{1}{64 \pi^{2}} \sum_{i}(-1)^{F} n_{i} M_{i}^{4}(h)\left[\log \frac{M_{i}^{2}(h)}{\mu^{2}}-c_{i}\right],
$$

where the summation is over all the fields interacting with the $h$-field. $F=0,1$ for bosons and fermions respectively, $n_{i}$ is the total of degrees of freedom of the particle and $M_{i}^{2}$ are the field-dependent mass terms

$$
M_{i}^{2}(h)=\kappa_{i} h^{2}-\kappa_{i}^{\prime}
$$

with the corresponing coefficients are shown in table 1 in the last column. Note that the massless fileds do not contribute to eq. (4.9), and to eq. (4.8). Hence, we only include top quark from SM, and the other contributions are neglected. For Type-III+ISS, their contributions comes after the mass threshold $M_{N_{i}}$. The full effective potential can be read from eq. (4.7), where each of these extra one-loop terms can be explained from eq. (4.8). The effective poential with only SM like Higgs field can be explained as eq. (4.6) with the effective coupling given as follows:

$$
\begin{aligned}
& \lambda_{\text {eff }}(h, \mu) \simeq \underbrace{\lambda_{h}(\mu)}_{\text {tree-level }}+\frac{1}{16 \pi^{2}}\{\underbrace{\sum_{\substack{i=W^{ \pm}, Z, t, h, G^{ \pm}, G^{0}}} n_{i} \kappa_{i}^{2}\left[\log \frac{\kappa_{i} h^{2}}{\mu^{2}}-c_{i}\right]}_{\text {Contribution from SM }}+\underbrace{\sum_{i=H, A, H^{ \pm}} n_{i} \kappa_{i}^{2}\left[\log \frac{\kappa_{i} h^{2}}{\mu^{2}}-c_{i}\right]}_{\text {Contribution from ID }} \\
&+ \underbrace{\left.2 \sum_{i=1,2} n_{i} \kappa_{i}^{2}\left[\log \frac{\kappa_{i} h^{2}}{\mu^{2}}-c_{i}\right]\right\} .}_{\text {Contribution from Type-III+ISS }}
\end{aligned}
$$




\begin{tabular}{|c|c|c|c|c|c|c|}
\hline Particles & $i$ & $F$ & $n_{i}$ & $c_{i}$ & $\kappa_{i}$ & $\kappa_{i}^{\prime}$ \\
\hline \hline \multirow{5}{*}{ SM } & $W^{ \pm}$ & 0 & 6 & $5 / 6$ & $g_{2}^{2} / 4$ & 0 \\
& $Z$ & 0 & 3 & $5 / 6$ & $\left(g_{1}^{2}+g_{2}^{2}\right) / 4$ & 0 \\
& $t$ & 1 & 12 & $3 / 2$ & $Y_{t}^{2}$ & 0 \\
& $h$ & 0 & 1 & $3 / 2$ & $\lambda_{h}$ & $m_{1}^{2}$ \\
& $G^{ \pm}$ & 0 & 2 & $3 / 2$ & $\lambda_{h}$ & $m_{1}^{2}$ \\
& $G^{0}$ & 0 & 1 & $3 / 2$ & $\lambda_{h}$ & $m_{1}^{2}$ \\
\hline \multirow{5}{*}{ Inert } & $H^{ \pm}$ & 0 & 2 & $3 / 2$ & $\lambda_{3} / 2$ & $m_{2}^{2}$ \\
& $H$ & 0 & 1 & $3 / 2$ & $\left(\lambda_{3}+\lambda_{4}+2 \lambda_{5}\right) / 2$ & $m_{2}^{2}$ \\
& $A$ & 0 & 1 & $3 / 2$ & $\left(\lambda_{3}+\lambda_{4}-2 \lambda_{5}\right) / 2$ & $m_{2}^{2}$ \\
\hline Type3seesaw +ISS & $\Sigma_{1 i}$ & 1 & 2 & $3 / 2$ & $Y_{N}^{2} / 2$ & 0 \\
\hline
\end{tabular}

Table 1. Coefficients in the Coleman-Weinberg effective potential needed for the study, cf. eq. (4.8).

In the case of inverse seesaw in the limit $\mu_{\Sigma} \rightarrow 0$, the triplet fermion masses are doubledegenerate per generation. Thus we take an extra factor of two for each new Type-III fermion contribution in eq. (4.10). We then study the $\lambda_{\text {eff }}(h, \mu)$ in our model to distinguish the stability, metastability and instability regions. $h=\mu$ is taken for the numerical analysis as at this scale we have scale-invariant potential [122].

\subsection{Phase diagrams: stable, metastable and unstable regions}

For $\lambda_{\text {eff }} \geq 0$ the potential is bounded from below and the region is termed as the stable region. In this region we expect to have a global EW minimum. On the other hand, for $\lambda_{\text {eff }}<0$ the potential develops a second minima and there can be tunnelling to the global second minima sufficiently faster. But there exists regions when such tunnelling is not fast enough and EW minimum still can survive due to the tunnelling rate which is greater than the age of the Today's universe. Such possibilities are known as metastable vacuua. The tunnelling rate at zero temperature has been calculated as

$$
P=T_{0}^{4} \mu^{4} \exp \left[\frac{-8 \pi^{2}}{3 \lambda_{\mathrm{eff}}(\mu)}\right],
$$

where $T_{0}$ is time corresponds to the age of the universe, and $\mu$ is the scale with maximum probability, i.e. $\frac{\partial P}{\partial \mu}=0$. The $\lambda$ values at different scales can be obtained as:

$$
\lambda_{\text {eff }}(\mu)=\frac{\lambda_{\text {eff }}(v)}{1-\frac{3}{2 \pi^{2}} \log \left(\frac{v}{\mu}\right) \lambda_{\text {eff }}(v)},
$$

where $v \simeq 246 \mathrm{GeV}$. If we substitute $P=1, T=10^{10}$ years and $\mu=v$ in eq. (4.11), one finds $\lambda_{\text {eff }}(v)=0.0623$. Demanding $P<1$, for $T=10^{10}$ years old universe would estimate the condition that such tunnelling rates corresponds to the metastability as given by [6]:

$$
0>\lambda_{\text {eff }}(\mu) \gtrsim \frac{-0.065}{1-0.01 \log \left(\frac{v}{\mu}\right)} .
$$


The condition of $\lambda_{\text {eff }}<0$ but outside region eq. (4.13), corresponds to when potential develops a second minima as mentioned before and if the tunneling rate from the EW vacuum to this second vacuua is less than the age of the universe then it depicts the unstable region. It is evident from eq. (4.10), these different solutions are sensitive to the choices of the scale $\mu$ and other model parameters such as bare masses and couplings.

Figure 8 represents the phase diagrams in terms of Higgs boson mass and top quark pole mass in $\mathrm{GeV}$. Different regions of the solution spaces corresponding to the unstable, metastable and stable regions are depicted in red, yellow and green colours respectively. The contours describe the current experimental $1 \sigma, 2 \sigma, 3 \sigma$ values with the dot specifying the central value in the $\left(M_{h}, M_{t}\right)$ plane $[9,63]$. To obtain the regions we vary all the $\lambda_{i}=0.1-$ 0.8 while the $\lambda_{1}$ and $y_{t}$ are varied to attain the Higgs boson mass within $120-128 \mathrm{GeV}$ and top quark mass within $168-182 \mathrm{GeV}$ respectively. In figure 8 we fix $M_{N}=100 \mathrm{GeV}$ and vary $Y_{N}=0.1-0.4$ for two generations of SU(2) triplet fermions. Figure $8(\mathrm{a})$ and figure $8(\mathrm{~b})$ present the scenarios with ID+Type-III+ISS for relatively lower values of $Y_{N}=0.1,0.3$. It is realized that the scenarios are stable till Planck scale.

As shown in figure $8(\mathrm{a})$, in this scenario, $\lambda_{\text {eff }}$ becomes more positive and the region is fully in the stable region till Planck scale. This occurs, as there is more positive contribution from $g_{2}$ compared to negative effect from fermions for lower values of $Y_{N}$. Additionally, the inert doublet also adds more scalars to the effective potential, leading to the enhanced stability. In figure $8(\mathrm{~b})$ we depict the scenario for $Y_{N}=0.3$, where negative fermionic effect starts showing up which is compensated by the scalar effect of IDM. As is evident, the stability is still more than SM, and hence, the $3 \sigma$ contour in $m_{h}-m_{t}$ plane just touches the region of metastability. Further enhancement in the value of $Y_{N}$ counters the positive scalar effect of IDM, and for $Y_{N}=0.4$ the $2 \sigma$ region enters in the unstable region as described in figure $8(\mathrm{c})$.

Figure $8(\mathrm{~d})$ describes the scenario for Type-III seesaw with two generations of triplet fermions, assuming $Y_{N}=0.4$. It can be seen that the whole region is unstable. Further addition of ISS SU $(2)_{L}$ triplet fermions which directly do not give negative contributions but enhance $g_{2}$ to more positive value as discussed before leads to Type-III seesaw+ISS scenario marginally extending into the metastable region for $Y_{N}=0.4$, as depicted in figure 8(e). Instead of ISS fermions addition of inert doublet also have the similar effect and pushes the potential into the metastable region as shown in figure 8(f). This further motivates the extension of Type-III seesaw scenario with ISS and IDM to achieve the stability at larger values of $Y_{N}$ as described in figure $8(\mathrm{~d})$.

Figure 9 we increase $M_{N}=10^{3} \mathrm{GeV}$ and analyse the phase diagrams as before. Due to this enhancement in $\mathrm{SU}(2)_{L}$ triplet fermion mass their negative loop effects will now be reduced. This can be realised from figures $9(\mathrm{a})-9(\mathrm{~b})$, where $\lambda_{\text {eff }}$ is highly positive and the regions are fully stable as compared to figures $8(\mathrm{a})-8(\mathrm{~b})$. As we increase $Y_{N}=0.4$, the negative fermionic effect starts showing up in figure 9 (c). The region now lies in the metastable region, however touching the stable region, contrary to figure 8 (c) where some part is in unstable region.

Figure $9(\mathrm{~d})$ describes the only Type-III scenario for $Y_{N}=0.4$ in which the central value in the $\left(M_{h}, M_{t}\right)$ plane lies in the unstable region similar to figure $8(\mathrm{~d})$. An extension 


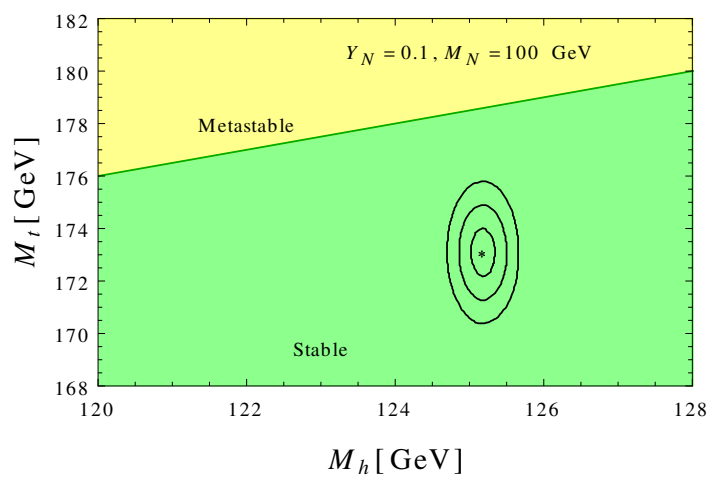

(a) ID+Type-III+ISS

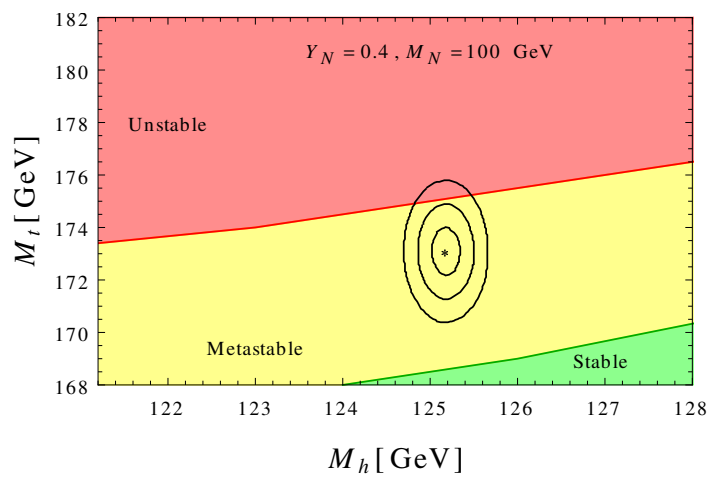

(c) ID+Type-III+ISS

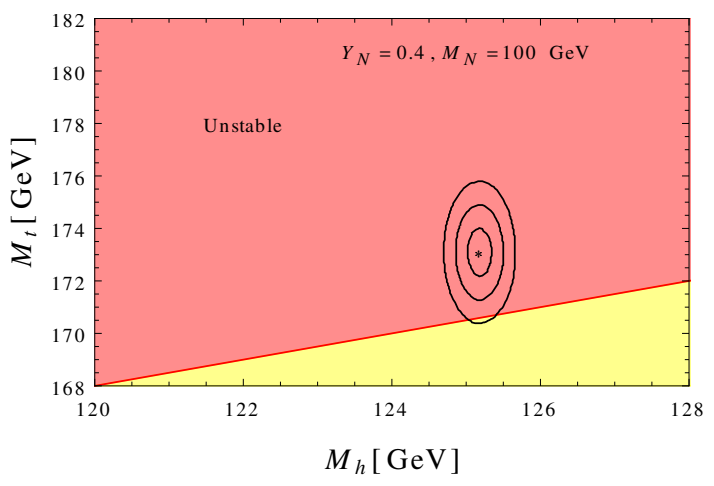

(e) Type-III+ISS

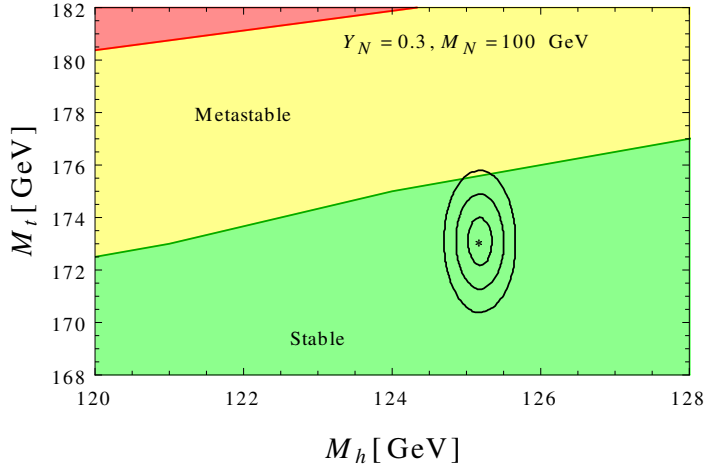

(b) ID+Type-III+ISS

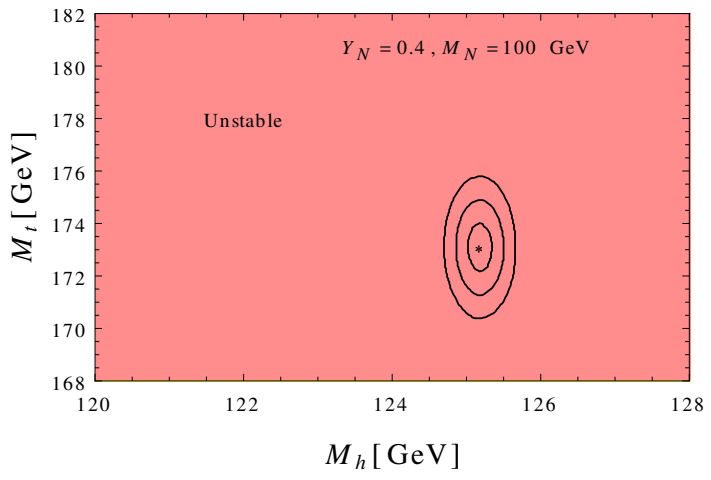

(d) Type-III

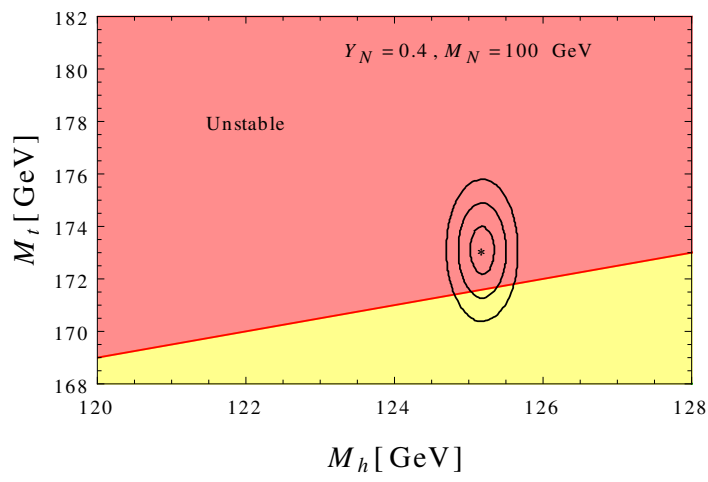

(f) ID+Type-III

Figure 8. Phase diagram in terms of Higgs and top pole masses in $\mathrm{GeV}$ with $M_{N}=100 \mathrm{GeV}$ in figure 8(a), 8(b), 8(c) for ID+Type-III+ISS scenarios with $Y_{N}=0.1,0.3,0.4$ respectively. Figure 8(d) is for Type-III only and figures $8(\mathrm{e}), 8(\mathrm{f})$ are for Type-III+ISS with $Y_{N}=0.4$. The unstable, metastable and stable regions are depicted by the colours red, yellow and green respectively. The contours describe the current experimental $1 \sigma, 2 \sigma, 3 \sigma$ values with the dot specifying the central value in the $\left(M_{h}, M_{t}\right)$ plane.

of Type-III seesaw with ISS in figure 9(e) and an extension of Type-III seesaw with inert doublet in figure $9(\mathrm{f})$ moves the potential into the metastable region similar to $M_{N}=$ $100 \mathrm{GeV}$ case in figures $8(\mathrm{e})-8(\mathrm{f})$ respectively. 


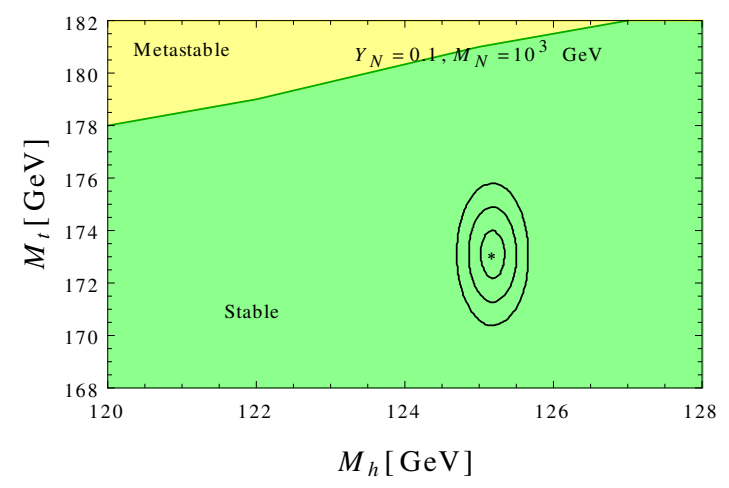

(a) ID+Type-III+ISS

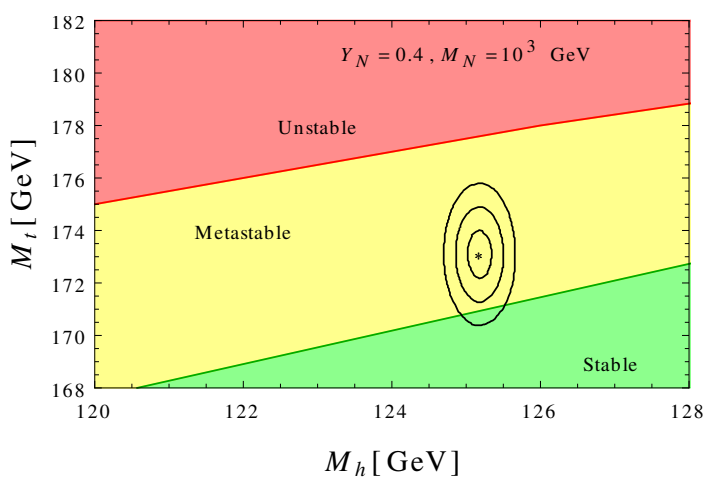

(c) ID+Type-III+ISS

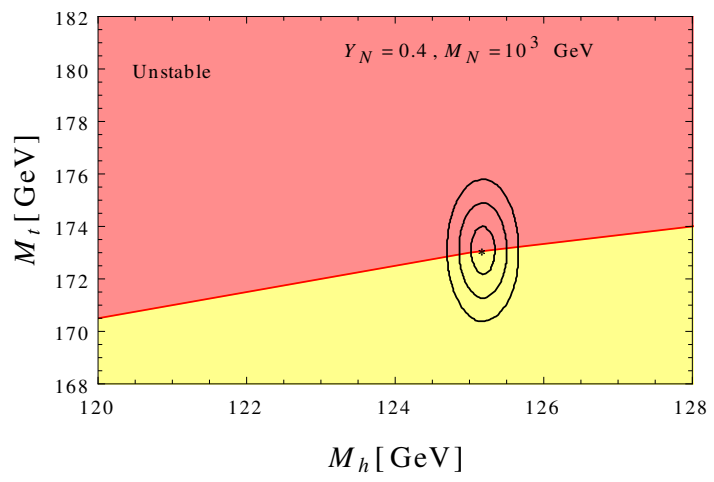

(e) Type-III+ISS

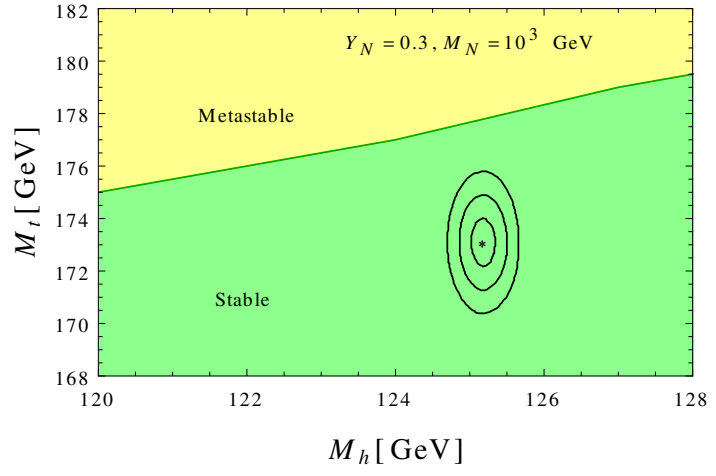

(b) ID+Type-III+ISS

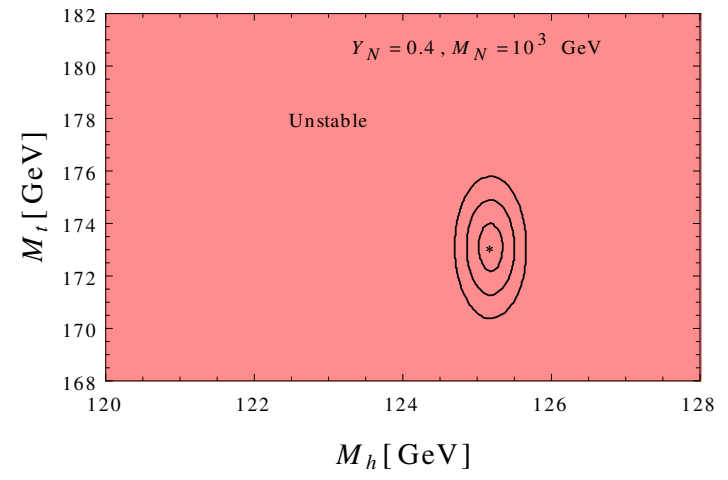

(d) Type-III

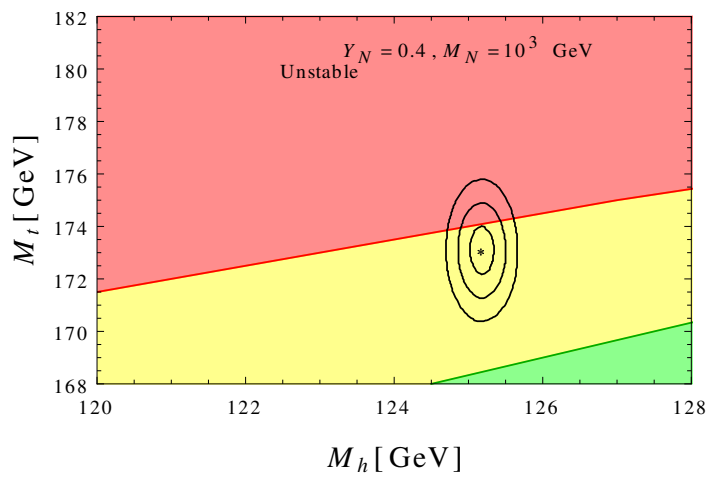

(f) ID+Type-III

Figure 9. Phase diagram in terms of Higgs and top pole masses in $\mathrm{GeV}$ with $M_{N}=10^{3} \mathrm{GeV}$ in figures 9(a), 9(b), 9(c) for ID+Type-III+ISS scenarios with $Y_{N}=0.1,0.3,0.4$ respectively. Figure $9(\mathrm{~d})$ is for Type-III only and figures $9(\mathrm{e}), 9(\mathrm{f})$ are for Type-III+ISS with $Y_{N}=0.4$. The unstable, metastable and stable regions are depicted by the colours red, yellow and green respectively. The contours describe the current experimental $1 \sigma, 2 \sigma, 3 \sigma$ values with the dot specifying the central value in the $\left(M_{h}, M_{t}\right)$ plane.

The inert Higgs doublet model provides a dark matter in terms of either $A$ or $H_{0}$ whichever is lighter $[38,54,67]$. Being $\mathrm{SU}(2)$ doublet the dominant mode of annihilation is $\mathrm{DMDM} \rightarrow \mathrm{W}^{ \pm} \mathrm{W}^{\mp}$ with sub-dominant annihilation and co-annihilation modes of $Z Z$ and 
$Z W^{ \pm}$depends on the gauge couplings for $M_{A}>M_{h}$. It was shown that correct DM relic puts a lower bounds on the DM mass of $700 \mathrm{GeV}$ and eq. (2.6) implies $m_{22} \gtrsim 665 \mathrm{GeV}$. This is very crucial in terms of figure 8 and figure 9 as we vary $\lambda_{\mathrm{i}}(\mathrm{EW})=0.01-0.8$ while the $\lambda_{1}$ and $y_{t}$ are varied to attain the Higgs boson mass within $120-128 \mathrm{GeV}$ and top quark mass within $168-182 \mathrm{GeV}$. However, the RGE evolution of the quartic couplings that we use in figure 8 and 9 is independent of the value of $m_{22}$. Since, the mass spectra of $H_{0}$ and $A$ depend on the $m_{22}$ and $\lambda_{345}\left(=\lambda_{3}+\lambda_{4}-2 \lambda_{5}\right)$ the quartic coupling combination (see eq. (2.6)) and we can always choose $m_{22}$ in such a way that the DM mass will satisfy the correct DM relic density. Thus above conclusions are consistent with the DM relic bounds.

Similarly, the EW value of $\mu_{\Sigma}$ will not effect the RGE evolution of the quartic couplings. Figure 8 and 9 fix $M_{N}$ as $100 \mathrm{GeV}$ and $1000 \mathrm{GeV}$ for three diffrent fixed values of $Y_{N}$. Still, we can choose $\mu_{\Sigma}$ in such a way to satisfy the $\mathrm{eV}$ order light neutrino mass (see eq. (2.16)). Thus the chosen benchmark points can satisfy both DM mass compatible with correct relic density and $\mathrm{eV}$ scale neutrino mass.

\section{Discussions and conclusion}

In this article we studied the vacuum stability of the electroweak vacuum in the presence of Type-III fermions along with inverse seesaw fermions. Unlike Type-I seesaw case, Type-III fermions are in the triplet representation of $\mathrm{SU}(2)$ which contribute to the beta-function of the gauge coupling $g_{2}$ even at one-loop level. This is a positive effect and increases from Type-III to Type-III+ISS+ID case step wise and makes the $g_{2}$ grow to higher values as scale increases. $g_{2}$ certainly becomes not perturbative below Planck scale or GUT scale for three generations of Type-III fermions. It is only with the two generation that we are able to acquire the Planck or GUT scale stability.

The enhancement of $g_{2}$ also has impact on the scalar quartic couplings which makes them non-perturbative much before compared to SM or SM+Type-I+ISS [54]. For lower values of quartic couplings the $g_{2}$ effect is the dominant. At larger values of $\lambda_{i}$ (except the $\lambda_{1}$ which is fixed by the Higgs mass at EW scale) $\lambda_{\mathrm{i}} \operatorname{Tr}\left(\mathrm{Y}_{\mathrm{N}}^{\dagger} \mathrm{Y}_{\mathrm{N}}\right)$ effect creeps in making the quartic couplings further divergent. However, a further increment of $Y_{N}$ will bring down the stability bound by pushing $\lambda_{1}$ to negative direction which is proportional to $\operatorname{Tr}\left(\mathrm{Y}_{\mathrm{N}}^{\dagger} \mathrm{Y}_{\mathrm{N}} \mathrm{Y}_{\mathrm{N}}^{\dagger} \mathrm{Y}_{\mathrm{N}}\right)$. For Planck scale perturbativity we can go up to $\lambda_{5} \sim 0.17$ with $\lambda_{1}=$ 0.126 and other $\lambda_{i}=0.10$ at the EW scale for $Y_{N}=0.40$. The effective potential approach calculations show that even for $Y_{N}=0.30$ for $m_{N}=100,1000 \mathrm{GeV}$ the model ID+TypeIII+ISS with two generations of new fermions lies in the stable region for lower values of $Y_{N}$ and draws to metastable region for higher values of $Y_{N}$. However, only Type-III scenario belongs to unstable regions in both cases, whereas ID+Type-III and Type-III+ISS scenarios can be in between metastable and unstable regions.

IDM is generally motivated to provide the much needed DM to explain the DM relic and other experimental observations. Nevertheless, it is also supported to enhance the stability of electroweak vacuum. Being in $Z_{2}$-odd multiplet it does not couple to the SU(2) triplet fermions which makes their phenomenology more illusive. No two-body decays are allowed for the Type-III fermions into any of the inert Higgs bosons. In [38, 54] authors 
have shown that due to compressed spectrum only some three- and four-body decays are allowed maintaining the $Z_{2}$ symmetry of the Lagrangian. In [37, 38, 123-126] a detailed relic calculations has been carried out including the direct, indirect DM searches and collider phenomenology. It has been found out that the lightest $Z_{2}$-odd particles should be heavier than $700 \mathrm{GeV}$ in ID and $1176 \mathrm{GeV}$ in IT to satisfy the DM relic constraints which can be in the desired range to explain the AMS-02 positron excess observation $[38,127]$. The decays of charged Higgs boson can give rise to mono-lepton plus missing energy signatures [38] which can be isolated from displaced mono-leptonic signatures in real scalar and complex triplet scenarios [38, 128] and other charged Higgs signatures [118, 129-131]. Similar displaced charged leptonic signatures can be observed in the models with Type-I seesaw [118, 132-137] and Type-III seesaw [82, 138, 139].

The triplet fermions are searched a the LHC at $13 \mathrm{TeV}$ centre of mass energy with democratic branching fractions [140] and a lower bound of $620-840 \mathrm{GeV}$ has been put at $2 \sigma$ level. However, due the presence and mixing with other set of $\mathrm{SU}(2)$ triplet fermions involving in inverse seesaw and non-democratic branching can substantially reduce the mass limit allowing even smaller triplet fermion mass. Type-III fermions can also be looked via their angular distributions at the LHC [91].

\section{Acknowledgments}

SJ thanks DST/INSPIRES/03/2018/001207 for the financial support towards the PhD program. SJ also wants to thank Dr. Anirban Karan for useful discussions. PB wants to thank SERB CORE Grant CRG/2018/004971, MTR/2020/000668, Anomalies 2019IUSSTF and Anomalies 2020 for the support. PB thanks Prof. Debajyoti Chaudhury for useful discussion. PB and SJ also want to thank Mr. Saiyad Ashanujjaman for the help in SARAH. PB and SJ also thank IOPB for the visit and local hospitality in the early part of the project.

\section{A Two-loop $\beta$-functions with two generations}

\section{A.1 Scalar quartic couplings}

$$
\begin{aligned}
\beta_{\lambda_{1}}= & \frac{1}{16 \pi^{2}}\left[\frac{27}{200} g_{1}^{4}+\frac{9}{20} g_{1}^{2} g_{2}^{2}+\frac{9}{8} g_{2}^{4}-\frac{9}{5} g_{1}^{2} \lambda_{1}-9 g_{2}^{2} \lambda_{1}+24 \lambda_{1}^{2}+2 \lambda_{3}^{2}+2 \lambda_{3} \lambda_{4}+\lambda_{4}^{2}+4 \lambda_{5}^{2}\right. \\
& +12 \lambda_{1} \operatorname{Tr}\left(Y_{d} Y_{d}^{\dagger}\right)+4 \lambda_{1} \operatorname{Tr}\left(Y_{e} Y_{e}^{\dagger}\right)+12 \lambda_{1} \operatorname{Tr}\left(Y_{N} Y_{N}^{\dagger}\right)+12 \lambda_{1} \operatorname{Tr}\left(Y_{u} Y_{u}^{\dagger}\right)-6 \operatorname{Tr}\left(Y_{d} Y_{d}^{\dagger} Y_{d} Y_{d}^{\dagger}\right) \\
& \left.-2 \operatorname{Tr}\left(Y_{e} Y_{e}^{\dagger} Y_{e} Y_{e}^{\dagger}\right)-8 \operatorname{Tr}\left(Y_{e} Y_{N}^{\dagger} Y_{N} Y_{e}^{\dagger}\right)-10 \operatorname{Tr}\left(Y_{N} Y_{N}^{\dagger} Y_{N} Y_{N}^{\dagger}\right)-6 \operatorname{Tr}\left(Y_{u} Y_{u}^{\dagger} Y_{u} Y_{u}^{\dagger}\right)\right] \\
& +\frac{1}{\left(16 \pi^{2}\right)^{2}}\left[-\frac{3537}{2000} g_{1}^{6}-\frac{1719}{400} g_{1}^{4} g_{2}^{2}-\frac{559}{80} g_{1}^{2} g_{2}^{4}+\frac{35}{16} g_{2}^{6}+\frac{1953}{200} g_{1}^{4} \lambda_{1}+\frac{117}{20} g_{1}^{2} g_{2}^{2} \lambda_{1}+\frac{269}{8} g_{2}^{4} \lambda_{1}\right. \\
& +108 g_{2}^{2} \lambda_{1}^{2}-312 \lambda_{1}^{3}+\frac{9}{10} g_{1}^{4} \lambda_{3}+\frac{15}{2} g_{2}^{4} \lambda_{3}+\frac{12}{5} g_{1}^{2} \lambda_{3}^{2}+12 g_{2}^{2} \lambda_{3}^{2}-20 \lambda_{1} \lambda_{3}^{2}-8 \lambda_{3}^{3}+\frac{9}{20} g_{1}^{4} \lambda_{4} \\
& +\frac{3}{2} g_{1}^{2} g_{2}^{2} \lambda_{4}+\frac{15}{4} g_{2}^{4} \lambda_{4}+\frac{12}{5} g_{1}^{2} \lambda_{3} \lambda_{4}+12 g_{2}^{2} \lambda_{3} \lambda_{4}-20 \lambda_{1} \lambda_{3} \lambda_{4}-12 \lambda_{3}^{2} \lambda_{4}+\frac{6}{5} g_{1}^{2} \lambda_{4}^{2} \\
& +3 g_{2}^{2} \lambda_{4}^{2}-12 \lambda_{1} \lambda_{4}^{2}-16 \lambda_{3} \lambda_{4}^{2}-6 \lambda_{4}^{3}-\frac{12}{5} g_{1}^{2} \lambda_{5}^{2}-56 \lambda_{1} \lambda_{5}^{2}-80 \lambda_{3} \lambda_{5}^{2}-88 \lambda_{4} \lambda_{5}^{2}+\frac{108}{5} g_{1}^{2} \lambda_{1}^{2}
\end{aligned}
$$




$$
\begin{aligned}
& +\frac{1}{20}\left(-5\left(64 \lambda_{1}\left(-5 g_{3}^{2}+9 \lambda_{1}\right)-90 g_{2}^{2} \lambda_{1}+9 g_{2}^{4}\right)+9 g_{1}^{4}+g_{1}^{2}\left(50 \lambda_{1}+54 g_{2}^{2}\right)\right) \operatorname{Tr}\left(Y_{d} Y_{d}^{\dagger}\right) \\
& -\frac{3}{20}\left(15 g_{1}^{4}-2 g_{1}^{2}\left(11 g_{2}^{2}+25 \lambda_{1}\right)+5\left(-10 g_{2}^{2} \lambda_{1}+64 \lambda_{1}^{2}+g_{2}^{4}\right)\right) \operatorname{Tr}\left(Y_{e} Y_{e}^{\dagger}\right)-\frac{27}{100} g_{1}^{4} \operatorname{Tr}\left(Y_{N} Y_{N}^{\dagger}\right) \\
& -\frac{57}{10} g_{1}^{2} g_{2}^{2} \operatorname{Tr}\left(Y_{N} Y_{N}^{\dagger}\right)+\frac{7}{4} g_{2}^{4} \operatorname{Tr}\left(Y_{N} Y_{N}^{\dagger}\right)+\frac{9}{2} g_{1}^{2} \lambda_{1} \operatorname{Tr}\left(Y_{N} Y_{N}^{\dagger}\right)+\frac{165}{2} g_{2}^{2} \lambda_{1} \operatorname{Tr}\left(Y_{N} Y_{N}^{\dagger}\right) \\
& -144 \lambda_{1}^{2} \operatorname{Tr}\left(Y_{N} Y_{N}^{\dagger}\right)-\frac{171}{100} g_{1}^{4} \operatorname{Tr}\left(Y_{u} Y_{u}^{\dagger}\right)+\frac{63}{10} g_{1}^{2} g_{2}^{2} \operatorname{Tr}\left(Y_{u} Y_{u}^{\dagger}\right)-\frac{9}{4} g_{2}^{4} \operatorname{Tr}\left(Y_{u} Y_{u}^{\dagger}\right) \\
& +\frac{17}{2} g_{1}^{2} \lambda_{1} \operatorname{Tr}\left(Y_{u} Y_{u}^{\dagger}\right)+\frac{45}{2} g_{2}^{2} \lambda_{1} \operatorname{Tr}\left(Y_{u} Y_{u}^{\dagger}\right)+80 g_{3}^{2} \lambda_{1} \operatorname{Tr}\left(Y_{u} Y_{u}^{\dagger}\right)-144 \lambda_{1}^{2} \operatorname{Tr}\left(Y_{u} Y_{u}^{\dagger}\right) \\
& +\frac{4}{5} g_{1}^{2} \operatorname{Tr}\left(Y_{d} Y_{d}^{\dagger} Y_{d} Y_{d}^{\dagger}\right)-32 g_{3}^{2} \operatorname{Tr}\left(Y_{d} Y_{d}^{\dagger} Y_{d} Y_{d}^{\dagger}\right)-3 \lambda_{1} \operatorname{Tr}\left(Y_{d} Y_{d}^{\dagger} Y_{d} Y_{d}^{\dagger}\right)-42 \lambda_{1} \operatorname{Tr}\left(Y_{d} Y_{u}^{\dagger} Y_{u} Y_{d}^{\dagger}\right) \\
& -\frac{12}{5} g_{1}^{2} \operatorname{Tr}\left(Y_{e} Y_{e}^{\dagger} Y_{e} Y_{e}^{\dagger}\right)-\lambda_{1} \operatorname{Tr}\left(Y_{e} Y_{e}^{\dagger} Y_{e} Y_{e}^{\dagger}\right)-\frac{24}{5} g_{1}^{2} \operatorname{Tr}\left(Y_{e} Y_{N}^{\dagger} Y_{N} Y_{e}^{\dagger}\right)+30 \operatorname{Tr}\left(Y_{u} Y_{u}^{\dagger} Y_{u} Y_{u}^{\dagger} Y_{u} Y_{u}^{\dagger}\right) \\
& -16 g_{2}^{2} \operatorname{Tr}\left(Y_{e} Y_{N}^{\dagger} Y_{N} Y_{e}^{\dagger}\right)+38 \lambda_{1} \operatorname{Tr}\left(Y_{e} Y_{N}^{\dagger} Y_{N} Y_{e}^{\dagger}\right)-40 g_{2}^{2} \operatorname{Tr}\left(Y_{N} Y_{N}^{\dagger} Y_{N} Y_{N}^{\dagger}\right) \\
& -5 \lambda_{1} \operatorname{Tr}\left(Y_{N} Y_{N}^{\dagger} Y_{N} Y_{N}^{\dagger}\right)-\frac{8}{5} g_{1}^{2} \operatorname{Tr}\left(Y_{u} Y_{u}^{\dagger} Y_{u} Y_{u}^{\dagger}\right)-32 g_{3}^{2} \operatorname{Tr}\left(Y_{u} Y_{u}^{\dagger} Y_{u} Y_{u}^{\dagger}\right)-3 \lambda_{1} \operatorname{Tr}\left(Y_{u} Y_{u}^{\dagger} Y_{u} Y_{u}^{\dagger}\right) \\
& +30 \operatorname{Tr}\left(Y_{d} Y_{d}^{\dagger} Y_{d} Y_{d}^{\dagger} Y_{d} Y_{d}^{\dagger}\right)-12 \operatorname{Tr}\left(Y_{d} Y_{d}^{\dagger} Y_{d} Y_{u}^{\dagger} Y_{u} Y_{d}^{\dagger}\right)+6 \operatorname{Tr}\left(Y_{d} Y_{u}^{\dagger} Y_{u} Y_{d}^{\dagger} Y_{d} Y_{d}^{\dagger}\right) \\
& -6 \operatorname{Tr}\left(Y_{d} Y_{u}^{\dagger} Y_{u} Y_{u}^{\dagger} Y_{u} Y_{d}^{\dagger}\right)+10 \operatorname{Tr}\left(Y_{e} Y_{e}^{\dagger} Y_{e} Y_{e}^{\dagger} Y_{e} Y_{e}^{\dagger}\right)+36 \operatorname{Tr}\left(Y_{e} Y_{e}^{\dagger} Y_{e} Y_{N}^{\dagger} Y_{N} Y_{e}^{\dagger}\right) \\
& \left.+38 \operatorname{Tr}\left(Y_{e} Y_{N}^{\dagger} Y_{N} Y_{e}^{\dagger} Y_{e} Y_{e}^{\dagger}\right)+150 \operatorname{Tr}\left(Y_{e} Y_{N}^{\dagger} Y_{N} Y_{N}^{\dagger} Y_{N} Y_{e}^{\dagger}\right)+94 \operatorname{Tr}\left(Y_{N} Y_{N}^{\dagger} Y_{N} Y_{N}^{\dagger} Y_{N} Y_{N}^{\dagger}\right)\right] \text {. } \\
& \beta_{\lambda_{2}}=\frac{1}{16 \pi^{2}}\left[24 \lambda_{2}^{2}+2 \lambda_{3}^{2}+2 \lambda_{3} \lambda_{4}+4 \lambda_{5}^{2}-9 g_{2}^{2} \lambda_{2}+\frac{27}{200} g_{1}^{4}+\frac{9}{20} g_{1}^{2}\left(-4 \lambda_{2}+g_{2}^{2}\right)+\frac{9}{8} g_{2}^{4}+\lambda_{4}^{2}\right] \\
& +\frac{1}{\left(16 \pi^{2}\right)^{2}}\left[-\frac{3537}{2000} g_{1}^{6}-\frac{1719}{400} g_{1}^{4} g_{2}^{2}-\frac{559}{80} g_{1}^{2} g_{2}^{4}+\frac{35}{16} g_{2}^{6}+\frac{1953}{200} g_{1}^{4} \lambda_{2}+\frac{117}{20} g_{1}^{2} g_{2}^{2} \lambda_{2}+\frac{269}{8} g_{2}^{4} \lambda_{2}\right. \\
& +108 g_{2}^{2} \lambda_{2}^{2}-312 \lambda_{2}^{3}+\frac{9}{10} g_{1}^{4} \lambda_{3}+\frac{15}{2} g_{2}^{4} \lambda_{3}+\frac{12}{5} g_{1}^{2} \lambda_{3}^{2}+12 g_{2}^{2} \lambda_{3}^{2}-20 \lambda_{2} \lambda_{3}^{2}-8 \lambda_{3}^{3}+\frac{9}{20} g_{1}^{4} \lambda_{4} \\
& +\frac{3}{2} g_{1}^{2} g_{2}^{2} \lambda_{4}+\frac{15}{4} g_{2}^{4} \lambda_{4}+\frac{12}{5} g_{1}^{2} \lambda_{3} \lambda_{4}+12 g_{2}^{2} \lambda_{3} \lambda_{4}-20 \lambda_{2} \lambda_{3} \lambda_{4}-12 \lambda_{3}^{2} \lambda_{4}+\frac{6}{5} g_{1}^{2} \lambda_{4}^{2}+\frac{108}{5} g_{1}^{2} \lambda_{2}^{2} \\
& +3 g_{2}^{2} \lambda_{4}^{2}-12 \lambda_{2} \lambda_{4}^{2}-16 \lambda_{3} \lambda_{4}^{2}-6 \lambda_{4}^{3}-\frac{12}{5} g_{1}^{2} \lambda_{5}^{2}-56 \lambda_{2} \lambda_{5}^{2}-80 \lambda_{3} \lambda_{5}^{2}-88 \lambda_{4} \lambda_{5}^{2} \\
& -6\left(2 \lambda_{3}^{2}+2 \lambda_{3} \lambda_{4}+4 \lambda_{5}^{2}+\lambda_{4}^{2}\right) \operatorname{Tr}\left(Y_{d} Y_{d}^{\dagger}\right)-2\left(2 \lambda_{3}^{2}+2 \lambda_{3} \lambda_{4}+4 \lambda_{5}^{2}+\lambda_{4}^{2}\right) \operatorname{Tr}\left(Y_{e} Y_{e}^{\dagger}\right) \\
& -12 \lambda_{3}^{2} \operatorname{Tr}\left(Y_{N} Y_{N}^{\dagger}\right)-12 \lambda_{3} \lambda_{4} \operatorname{Tr}\left(Y_{N} Y_{N}^{\dagger}\right)-6 \lambda_{4}^{2} \operatorname{Tr}\left(Y_{N} Y_{N}^{\dagger}\right)-24 \lambda_{5}^{2} \operatorname{Tr}\left(Y_{N} Y_{N}^{\dagger}\right) \\
& \left.-12 \lambda_{3}^{2} \operatorname{Tr}\left(Y_{u} Y_{u}^{\dagger}\right)-12 \lambda_{3} \lambda_{4} \operatorname{Tr}\left(Y_{u} Y_{u}^{\dagger}\right)-6 \lambda_{4}^{2} \operatorname{Tr}\left(Y_{u} Y_{u}^{\dagger}\right)-24 \lambda_{5}^{2} \operatorname{Tr}\left(Y_{u} Y_{u}^{\dagger}\right)\right] \text {. } \\
& \beta_{\lambda_{3}}=\frac{1}{16 \pi^{2}}\left[\frac{27}{100} g_{1}^{4}-\frac{9}{10} g_{1}^{2} g_{2}^{2}+\frac{9}{4} g_{2}^{4}-\frac{9}{5} g_{1}^{2} \lambda_{3}-9 g_{2}^{2} \lambda_{3}+12 \lambda_{1} \lambda_{3}+12 \lambda_{2} \lambda_{3}+4 \lambda_{3}^{2}+4 \lambda_{1} \lambda_{4}+4 \lambda_{2} \lambda_{4}\right. \\
& \left.+2 \lambda_{4}^{2}+8 \lambda_{5}^{2}+6 \lambda_{3} \operatorname{Tr}\left(Y_{d} Y_{d}^{\dagger}\right)+2 \lambda_{3} \operatorname{Tr}\left(Y_{e} Y_{e}^{\dagger}\right)+6 \lambda_{3} \operatorname{Tr}\left(Y_{N} Y_{N}^{\dagger}\right)+6 \lambda_{3} \operatorname{Tr}\left(Y_{u} Y_{u}^{\dagger}\right)\right] \\
& +\frac{1}{\left(16 \pi^{2}\right)^{2}}\left[-\frac{3537}{1000} g_{1}^{6}+\frac{909}{200} g_{1}^{4} g_{2}^{2}+\frac{289}{40} g_{1}^{2} g_{2}^{4}+\frac{35}{8} g_{2}^{6}+\frac{27}{10} g_{1}^{4} \lambda_{1}-3 g_{1}^{2} g_{2}^{2} \lambda_{1}+\frac{45}{2} g_{2}^{4} \lambda_{1}+\frac{27}{10} g_{1}^{4} \lambda_{2}\right. \\
& -3 g_{1}^{2} g_{2}^{2} \lambda_{2}+\frac{45}{2} g_{2}^{4} \lambda_{2}+\frac{1773}{200} g_{1}^{4} \lambda_{3}+\frac{33}{20} g_{1}^{2} g_{2}^{2} \lambda_{3}+\frac{209}{8} g_{2}^{4} \lambda_{3}+\frac{72}{5} g_{1}^{2} \lambda_{1} \lambda_{3}+72 g_{2}^{2} \lambda_{1} \lambda_{3} \\
& -60 \lambda_{1}^{2} \lambda_{3}+\frac{72}{5} g_{1}^{2} \lambda_{2} \lambda_{3}+72 g_{2}^{2} \lambda_{2} \lambda_{3}-60 \lambda_{2}^{2} \lambda_{3}+\frac{6}{5} g_{1}^{2} \lambda_{3}^{2}+6 g_{2}^{2} \lambda_{3}^{2}-72 \lambda_{1} \lambda_{3}^{2}-72 \lambda_{2} \lambda_{3}^{2} \\
& -12 \lambda_{3}^{3}+\frac{9}{10} g_{1}^{4} \lambda_{4}-\frac{9}{5} g_{1}^{2} g_{2}^{2} \lambda_{4}+\frac{15}{2} g_{2}^{4} \lambda_{4}+\frac{24}{5} g_{1}^{2} \lambda_{1} \lambda_{4}+36 g_{2}^{2} \lambda_{1} \lambda_{4}-16 \lambda_{1}^{2} \lambda_{4}+\frac{24}{5} g_{1}^{2} \lambda_{2} \lambda_{4} \\
& +36 g_{2}^{2} \lambda_{2} \lambda_{4}-16 \lambda_{2}^{2} \lambda_{4}-12 g_{2}^{2} \lambda_{3} \lambda_{4}-32 \lambda_{1} \lambda_{3} \lambda_{4}-32 \lambda_{2} \lambda_{3} \lambda_{4}-4 \lambda_{3}^{2} \lambda_{4}-\frac{6}{5} g_{1}^{2} \lambda_{4}^{2}
\end{aligned}
$$




$$
\begin{aligned}
& +6 g_{2}^{2} \lambda_{4}^{2}-28 \lambda_{1} \lambda_{4}^{2}-28 \lambda_{2} \lambda_{4}^{2}-16 \lambda_{3} \lambda_{4}^{2}-12 \lambda_{4}^{3}+\frac{48}{5} g_{1}^{2} \lambda_{5}^{2}-144 \lambda_{1} \lambda_{5}^{2}-144 \lambda_{2} \lambda_{5}^{2} \\
& -72 \lambda_{3} \lambda_{5}^{2}-176 \lambda_{4} \lambda_{5}^{2}+\frac{1}{20}\left(-5\left(-45 g_{2}^{2} \lambda_{3}+8\left(-20 g_{3}^{2} \lambda_{3}+3\left(2 \lambda_{3}^{2}+4 \lambda_{1}\left(3 \lambda_{3}+\lambda_{4}\right)\right.\right.\right.\right. \\
& \left.\left.\left.\left.+4 \lambda_{5}^{2}+\lambda_{4}^{2}\right)\right)+9 g_{2}^{4}\right)+9 g_{1}^{4}+g_{1}^{2}\left(25 \lambda_{3}-54 g_{2}^{2}\right)\right) \operatorname{Tr}\left(Y_{d} Y_{d}^{\dagger}\right)-\frac{1}{20}\left(45 g_{1}^{4}\right. \\
& \left.+5\left(-15 g_{2}^{2} \lambda_{3}+3 g_{2}^{4}+8\left(2 \lambda_{3}^{2}+4 \lambda_{1}\left(3 \lambda_{3}+\lambda_{4}\right)+4 \lambda_{5}^{2}+\lambda_{4}^{2}\right)\right)+g_{1}^{2}\left(66 g_{2}^{2}-75 \lambda_{3}\right)\right) \operatorname{Tr}\left(Y_{e} Y_{e}^{\dagger}\right) \\
& -\frac{27}{100} g_{1}^{4} \operatorname{Tr}\left(Y_{N} Y_{N}^{\dagger}\right)+\frac{57}{10} g_{1}^{2} g_{2}^{2} \operatorname{Tr}\left(Y_{N} Y_{N}^{\dagger}\right)+\frac{7}{4} g_{2}^{4} \operatorname{Tr}\left(Y_{N} Y_{N}^{\dagger}\right)+\frac{9}{4} g_{1}^{2} \lambda_{3} \operatorname{Tr}\left(Y_{N} Y_{N}^{\dagger}\right) \\
& +\frac{165}{4} g_{2}^{2} \lambda_{3} \operatorname{Tr}\left(Y_{N} Y_{N}^{\dagger}\right)-72 \lambda_{1} \lambda_{3} \operatorname{Tr}\left(Y_{N} Y_{N}^{\dagger}\right)-12 \lambda_{3}^{2} \operatorname{Tr}\left(Y_{N} Y_{N}^{\dagger}\right)-24 \lambda_{1} \lambda_{4} \operatorname{Tr}\left(Y_{N} Y_{N}^{\dagger}\right) \\
& -6 \lambda_{4}^{2} \operatorname{Tr}\left(Y_{N} Y_{N}^{\dagger}\right)-24 \lambda_{5}^{2} \operatorname{Tr}\left(Y_{N} Y_{N}^{\dagger}\right)-\frac{171}{100} g_{1}^{4} \operatorname{Tr}\left(Y_{u} Y_{u}^{\dagger}\right)-\frac{63}{10} g_{1}^{2} g_{2}^{2} \operatorname{Tr}\left(Y_{u} Y_{u}^{\dagger}\right) \\
& -\frac{9}{4} g_{2}^{4} \operatorname{Tr}\left(Y_{u} Y_{u}^{\dagger}\right)+\frac{17}{4} g_{1}^{2} \lambda_{3} \operatorname{Tr}\left(Y_{u} Y_{u}^{\dagger}\right)+\frac{45}{4} g_{2}^{2} \lambda_{3} \operatorname{Tr}\left(Y_{u} Y_{u}^{\dagger}\right)+40 g_{3}^{2} \lambda_{3} \operatorname{Tr}\left(Y_{u} Y_{u}^{\dagger}\right) \\
& -72 \lambda_{1} \lambda_{3} \operatorname{Tr}\left(Y_{u} Y_{u}^{\dagger}\right)-12 \lambda_{3}^{2} \operatorname{Tr}\left(Y_{u} Y_{u}^{\dagger}\right)-24 \lambda_{1} \lambda_{4} \operatorname{Tr}\left(Y_{u} Y_{u}^{\dagger}\right)-6 \lambda_{4}^{2} \operatorname{Tr}\left(Y_{u} Y_{u}^{\dagger}\right) \\
& -24 \lambda_{5}^{2} \operatorname{Tr}\left(Y_{u} Y_{u}^{\dagger}\right)-\frac{27}{2} \lambda_{3} \operatorname{Tr}\left(Y_{d} Y_{d}^{\dagger} Y_{d} Y_{d}^{\dagger}\right)-21 \lambda_{3} \operatorname{Tr}\left(Y_{d} Y_{u}^{\dagger} Y_{u} Y_{d}^{\dagger}\right)-24 \lambda_{4} \operatorname{Tr}\left(Y_{d} Y_{u}^{\dagger} Y_{u} Y_{d}^{\dagger}\right) \\
& -\frac{9}{2} \lambda_{3} \operatorname{Tr}\left(Y_{e} Y_{e}^{\dagger} Y_{e} Y_{e}^{\dagger}\right)+3 \lambda_{3} \operatorname{Tr}\left(Y_{e} Y_{N}^{\dagger} Y_{N} Y_{e}^{\dagger}\right)+8 \lambda_{4} \operatorname{Tr}\left(Y_{e} Y_{N}^{\dagger} Y_{N} Y_{e}^{\dagger}\right)-\frac{45}{2} \lambda_{3} \operatorname{Tr}\left(Y_{N} Y_{N}^{\dagger} Y_{N} Y_{N}^{\dagger}\right) \\
& \left.-\frac{27}{2} \lambda_{3} \operatorname{Tr}\left(Y_{u} Y_{u}^{\dagger} Y_{u} Y_{u}^{\dagger}\right)\right] \text {. } \\
& \beta_{\lambda_{4}}=\frac{1}{16 \pi^{2}}\left[\frac{9}{5} g_{1}^{2} g_{2}^{2}-\frac{9}{5} g_{1}^{2} \lambda_{4}-9 g_{2}^{2} \lambda_{4}+4 \lambda_{1} \lambda_{4}+4 \lambda_{2} \lambda_{4}+8 \lambda_{3} \lambda_{4}+4 \lambda_{4}^{2}+32 \lambda_{5}^{2}+6 \lambda_{4} \operatorname{Tr}\left(Y_{d} Y_{d}^{\dagger}\right)\right. \\
& \left.+2 \lambda_{4} \operatorname{Tr}\left(Y_{e} Y_{e}^{\dagger}\right)+6 \lambda_{4} \operatorname{Tr}\left(Y_{N} Y_{N}^{\dagger}\right)+6 \lambda_{4} \operatorname{Tr}\left(Y_{u} Y_{u}^{\dagger}\right)\right] \\
& +\frac{1}{\left(16 \pi^{2}\right)^{2}}\left[-\frac{657}{50} g_{1}^{4} g_{2}^{2}-\frac{106}{5} g_{1}^{2} g_{2}^{4}+6 g_{1}^{2} g_{2}^{2} \lambda_{1}+6 g_{1}^{2} g_{2}^{2} \lambda_{2}+\frac{6}{5} g_{1}^{2} g_{2}^{2} \lambda_{3}+\frac{1413}{200} g_{1}^{4} \lambda_{4}+\frac{153}{20} g_{1}^{2} g_{2}^{2} \lambda_{4}\right. \\
& +\frac{89}{8} g_{2}^{4} \lambda_{4}+\frac{24}{5} g_{1}^{2} \lambda_{1} \lambda_{4}-28 \lambda_{1}^{2} \lambda_{4}+\frac{24}{5} g_{1}^{2} \lambda_{2} \lambda_{4}-28 \lambda_{2}^{2} \lambda_{4}+\frac{12}{5} g_{1}^{2} \lambda_{3} \lambda_{4}+36 g_{2}^{2} \lambda_{3} \lambda_{4} \\
& -80 \lambda_{1} \lambda_{3} \lambda_{4}-80 \lambda_{2} \lambda_{3} \lambda_{4}-28 \lambda_{3}^{2} \lambda_{4}+\frac{24}{5} g_{1}^{2} \lambda_{4}^{2}+18 g_{2}^{2} \lambda_{4}^{2}-40 \lambda_{1} \lambda_{4}^{2}-40 \lambda_{2} \lambda_{4}^{2}-28 \lambda_{3} \lambda_{4}^{2} \\
& +\frac{192}{5} g_{1}^{2} \lambda_{5}^{2}+216 g_{2}^{2} \lambda_{5}^{2}-192 \lambda_{1} \lambda_{5}^{2}-192 \lambda_{2} \lambda_{5}^{2}-192 \lambda_{3} \lambda_{5}^{2}-104 \lambda_{4} \lambda_{5}^{2} \\
& +\left(4\left(10 g_{3}^{2} \lambda_{4}-3\left(2 \lambda_{1} \lambda_{4}+2 \lambda_{3} \lambda_{4}+8 \lambda_{5}^{2}+\lambda_{4}^{2}\right)\right)+\frac{45}{4} g_{2}^{2} \lambda_{4}+g_{1}^{2}\left(\frac{27}{5} g_{2}^{2}+\frac{5}{4} \lambda_{4}\right)\right) \operatorname{Tr}\left(Y_{d} Y_{d}^{\dagger}\right) \\
& +\left(-4\left(2 \lambda_{1} \lambda_{4}+2 \lambda_{3} \lambda_{4}+8 \lambda_{5}^{2}+\lambda_{4}^{2}\right)+\frac{15}{4} g_{2}^{2} \lambda_{4}+\frac{3}{20} g_{1}^{2}\left(25 \lambda_{4}+44 g_{2}^{2}\right)\right) \operatorname{Tr}\left(Y_{e} Y_{e}^{\dagger}\right) \\
& -\frac{57}{5} g_{1}^{2} g_{2}^{2} \operatorname{Tr}\left(Y_{N} Y_{N}^{\dagger}\right)+\frac{9}{4} g_{1}^{2} \lambda_{4} \operatorname{Tr}\left(Y_{N} Y_{N}^{\dagger}\right)+\frac{165}{4} g_{2}^{2} \lambda_{4} \operatorname{Tr}\left(Y_{N} Y_{N}^{\dagger}\right)-24 \lambda_{1} \lambda_{4} \operatorname{Tr}\left(Y_{N} Y_{N}^{\dagger}\right) \\
& -24 \lambda_{3} \lambda_{4} \operatorname{Tr}\left(Y_{N} Y_{N}^{\dagger}\right)-12 \lambda_{4}^{2} \operatorname{Tr}\left(Y_{N} Y_{N}^{\dagger}\right)-96 \lambda_{5}^{2} \operatorname{Tr}\left(Y_{N} Y_{N}^{\dagger}\right)+\frac{63}{5} g_{1}^{2} g_{2}^{2} \operatorname{Tr}\left(Y_{u} Y_{u}^{\dagger}\right) \\
& +\frac{17}{4} g_{1}^{2} \lambda_{4} \operatorname{Tr}\left(Y_{u} Y_{u}^{\dagger}\right)+\frac{45}{4} g_{2}^{2} \lambda_{4} \operatorname{Tr}\left(Y_{u} Y_{u}^{\dagger}\right)+40 g_{3}^{2} \lambda_{4} \operatorname{Tr}\left(Y_{u} Y_{u}^{\dagger}\right)-24 \lambda_{1} \lambda_{4} \operatorname{Tr}\left(Y_{u} Y_{u}^{\dagger}\right) \\
& -24 \lambda_{3} \lambda_{4} \operatorname{Tr}\left(Y_{u} Y_{u}^{\dagger}\right)-12 \lambda_{4}^{2} \operatorname{Tr}\left(Y_{u} Y_{u}^{\dagger}\right)-96 \lambda_{5}^{2} \operatorname{Tr}\left(Y_{u} Y_{u}^{\dagger}\right)-\frac{27}{2} \lambda_{4} \operatorname{Tr}\left(Y_{d} Y_{d}^{\dagger} Y_{d} Y_{d}^{\dagger}\right) \\
& +27 \lambda_{4} \operatorname{Tr}\left(Y_{d} Y_{u}^{\dagger} Y_{u} Y_{d}^{\dagger}\right)-\frac{9}{2} \lambda_{4} \operatorname{Tr}\left(Y_{e} Y_{e}^{\dagger} Y_{e} Y_{e}^{\dagger}\right)-13 \lambda_{4} \operatorname{Tr}\left(Y_{e} Y_{N}^{\dagger} Y_{N} Y_{e}^{\dagger}\right)-\frac{45}{2} \lambda_{4} \operatorname{Tr}\left(Y_{N} Y_{N}^{\dagger} Y_{N} Y_{N}^{\dagger}\right) \\
& \left.-\frac{27}{2} \lambda_{4} \operatorname{Tr}\left(Y_{u} Y_{u}^{\dagger} Y_{u} Y_{u}^{\dagger}\right)\right] \text {. }
\end{aligned}
$$




$$
\begin{aligned}
\beta_{\lambda_{5}}= & \frac{1}{16 \pi^{2}}\left[-\frac{9}{5} g_{1}^{2} \lambda_{5}-9 g_{2}^{2} \lambda_{5}+4 \lambda_{1} \lambda_{5}+4 \lambda_{2} \lambda_{5}+8 \lambda_{3} \lambda_{5}+12 \lambda_{4} \lambda_{5}+6 \lambda_{5} \operatorname{Tr}\left(Y_{d} Y_{d}^{\dagger}\right)+2 \lambda_{5} \operatorname{Tr}\left(Y_{e} Y_{e}^{\dagger}\right)\right. \\
& \left.+6 \lambda_{5} \operatorname{Tr}\left(Y_{N} Y_{N}^{\dagger}\right)+6 \lambda_{5} \operatorname{Tr}\left(Y_{u} Y_{u}^{\dagger}\right)\right] \\
& +\frac{1}{\left(16 \pi^{2}\right)^{2}}\left[\frac{1413}{200} g_{1}^{4} \lambda_{5}+\frac{57}{20} g_{1}^{2} g_{2}^{2} \lambda_{5}+\frac{89}{8} g_{2}^{4} \lambda_{5}-\frac{12}{5} g_{1}^{2} \lambda_{1} \lambda_{5}-28 \lambda_{1}^{2} \lambda_{5}-\frac{12}{5} g_{1}^{2} \lambda_{2} \lambda_{5}-28 \lambda_{2}^{2} \lambda_{5}\right. \\
& +\frac{48}{5} g_{1}^{2} \lambda_{3} \lambda_{5}+36 g_{2}^{2} \lambda_{3} \lambda_{5}-80 \lambda_{1} \lambda_{3} \lambda_{5}-80 \lambda_{2} \lambda_{3} \lambda_{5}-28 \lambda_{3}^{2} \lambda_{5}+\frac{72}{5} g_{1}^{2} \lambda_{4} \lambda_{5}+72 g_{2}^{2} \lambda_{4} \lambda_{5} \\
& -88 \lambda_{1} \lambda_{4} \lambda_{5}-88 \lambda_{2} \lambda_{4} \lambda_{5}-76 \lambda_{3} \lambda_{4} \lambda_{5}-32 \lambda_{4}^{2} \lambda_{5}+24 \lambda_{5}^{3} \\
& +\frac{1}{4}\left(16\left(10 g_{3}^{2}-6 \lambda_{1}-6 \lambda_{3}-9 \lambda_{4}\right)+45 g_{2}^{2}+5 g_{1}^{2}\right) \lambda_{5} \operatorname{Tr}\left(Y_{d} Y_{d}^{\dagger}\right) \\
& +\frac{1}{4}\left(15 g_{1}^{2}+15 g_{2}^{2}-16\left(2 \lambda_{1}+2 \lambda_{3}+3 \lambda_{4}\right)\right) \lambda_{5} \operatorname{Tr}\left(Y_{e} Y_{e}^{\dagger}\right)+\frac{9}{4} g_{1}^{2} \lambda_{5} \operatorname{Tr}\left(Y_{N} Y_{N}^{\dagger}\right)+\frac{165}{4} g_{2}^{2} \lambda_{5} \operatorname{Tr}\left(Y_{N} Y_{N}^{\dagger}\right) \\
& -24 \lambda_{1} \lambda_{5} \operatorname{Tr}\left(Y_{N} Y_{N}^{\dagger}\right)-24 \lambda_{3} \lambda_{5} \operatorname{Tr}\left(Y_{N} Y_{N}^{\dagger}\right)-36 \lambda_{4} \lambda_{5} \operatorname{Tr}\left(Y_{N} Y_{N}^{\dagger}\right)+\frac{17}{4} g_{1}^{2} \lambda_{5} \operatorname{Tr}\left(Y_{u} Y_{u}^{\dagger}\right) \\
& +\frac{45}{4} g_{2}^{2} \lambda_{5} \operatorname{Tr}\left(Y_{u} Y_{u}^{\dagger}\right)+40 g_{3}^{2} \lambda_{5} \operatorname{Tr}\left(Y_{u} Y_{u}^{\dagger}\right)-24 \lambda_{1} \lambda_{5} \operatorname{Tr}\left(Y_{u} Y_{u}^{\dagger}\right)-24 \lambda_{3} \lambda_{5} \operatorname{Tr}\left(Y_{u} Y_{u}^{\dagger}\right) \\
& -36 \lambda_{4} \lambda_{5} \operatorname{Tr}\left(Y_{u} Y_{u}^{\dagger}\right)-\frac{3}{2} \lambda_{5} \operatorname{Tr}\left(Y_{d} Y_{d}^{\dagger} Y_{d} Y_{d}^{\dagger}\right)+3 \lambda_{5} \operatorname{Tr}\left(Y_{d} Y_{u}^{\dagger} Y_{u} Y_{d}^{\dagger}\right)-\frac{1}{2} \lambda_{5} \operatorname{Tr}\left(Y_{e} Y_{e}^{\dagger} Y_{e} Y_{e}^{\dagger}\right) \\
& \left.-21 \lambda_{5} \operatorname{Tr}\left(Y_{e} Y_{N}^{\dagger} Y_{N} Y_{e}^{\dagger}\right)-\frac{5}{2} \lambda_{5} \operatorname{Tr}\left(Y_{N} Y_{N}^{\dagger} Y_{N} Y_{N}^{\dagger}\right)-\frac{3}{2} \lambda_{5} \operatorname{Tr}\left(Y_{u} Y_{u}^{\dagger} Y_{u} Y_{u}^{\dagger}\right)\right]
\end{aligned}
$$

\section{A.2 Yukawa coupling}

$$
\begin{aligned}
\beta_{Y_{u}}= & \frac{1}{16 \pi^{2}}\left[-\frac{3}{2}\left(-Y_{u} Y_{u}^{\dagger} Y_{u}+Y_{u} Y_{d}^{\dagger} Y_{d}\right)\right. \\
& \left.+Y_{u}\left(3 \operatorname{Tr}\left(Y_{d} Y_{d}^{\dagger}\right)+3 \operatorname{Tr}\left(Y_{N} Y_{N}^{\dagger}\right)+3 \operatorname{Tr}\left(Y_{u} Y_{u}^{\dagger}\right)-8 g_{3}^{2}-\frac{17}{20} g_{1}^{2}-\frac{9}{4} g_{2}^{2}+\operatorname{Tr}\left(Y_{e} Y_{e}^{\dagger}\right)\right)\right] \\
& +\frac{1}{\left(16 \pi^{2}\right)^{2}}\left[\frac { 1 } { 8 0 } \left(20\left(11 Y_{u} Y_{d}^{\dagger} Y_{d} Y_{d}^{\dagger} Y_{d}-4 Y_{u} Y_{u}^{\dagger} Y_{u} Y_{d}^{\dagger} Y_{d}+6 Y_{u} Y_{u}^{\dagger} Y_{u} Y_{u}^{\dagger} Y_{u}-Y_{u} Y_{d}^{\dagger} Y_{d} Y_{u}^{\dagger} Y_{u}\right)\right.\right. \\
& +Y_{u} Y_{u}^{\dagger} Y_{u}\left(1280 g_{3}^{2}-180 \operatorname{Tr}\left(Y_{e} Y_{e}^{\dagger}\right)+223 g_{1}^{2}-540 \operatorname{Tr}\left(Y_{d} Y_{d}^{\dagger}\right)-540 \operatorname{Tr}\left(Y_{N} Y_{N}^{\dagger}\right)-540 \operatorname{Tr}\left(Y_{u} Y_{u}^{\dagger}\right)\right. \\
& \left.+Y_{u} Y_{d}^{\dagger} Y_{d}\left(100 \operatorname{Tr}\left(Y_{e} Y_{e}^{\dagger}\right)-1280 g_{3}^{2}+300 \operatorname{Tr}\left(Y_{d} Y_{d}^{\dagger}\right)+300 \operatorname{Tr}\left(Y_{N} Y_{N}^{\dagger}\right)+300 \operatorname{Tr}\left(Y_{u} Y_{u}^{\dagger}\right)-43 g_{1}^{2}+45 g_{2}^{2}\right)\right) \\
& +Y_{u}\left(\frac{1267}{600} g_{1}^{4}-\frac{9}{20} g_{1}^{2} g_{2}^{2}-\frac{5}{4} g_{2}^{4}+\frac{19}{15} g_{1}^{2} g_{3}^{2}+9 g_{2}^{2} g_{3}^{2}-108 g_{3}^{4}+6 \lambda_{1}^{2}+\lambda_{3}^{2}+\lambda_{3} \lambda_{4}+\lambda_{4}^{2}+6 \lambda_{5}^{2}\right. \\
& +\frac{5}{8}\left(32 g_{3}^{2}+9 g_{2}^{2}+g_{1}^{2}\right) \operatorname{Tr}\left(Y_{d} Y_{d}^{\dagger}\right)+\frac{15}{8}\left(g_{1}^{2}+g_{2}^{2}\right) \operatorname{Tr}\left(Y_{e} Y_{e}^{\dagger}\right)+\frac{9}{8} g_{1}^{2} \operatorname{Tr}\left(Y_{N} Y_{N}^{\dagger}\right)+\frac{165}{8} g_{2}^{2} \operatorname{Tr}\left(Y_{N} Y_{N}^{\dagger}\right) \\
& +\frac{17}{8} g_{1}^{2} \operatorname{Tr}\left(Y_{u} Y_{u}^{\dagger}\right)+\frac{45}{8} g_{2}^{2} \operatorname{Tr}\left(Y_{u} Y_{u}^{\dagger}\right)+20 g_{3}^{2} \operatorname{Tr}\left(Y_{u} Y_{u}^{\dagger}\right)-\frac{27}{4} \operatorname{Tr}\left(Y_{d} Y_{d}^{\dagger} Y_{d} Y_{d}^{\dagger}\right) \\
& +\frac{3}{2} \operatorname{Tr}\left(Y_{d} Y_{u}^{\dagger} Y_{u} Y_{d}^{\dagger}\right)-\frac{9}{4} \operatorname{Tr}\left(Y_{e} Y_{e}^{\dagger} Y_{e} Y_{e}^{\dagger}\right)-\frac{21}{2} \operatorname{Tr}\left(Y_{e} Y_{N}^{\dagger} Y_{N} Y_{e}^{\dagger}\right)-\frac{45}{4} \operatorname{Tr}\left(Y_{N} Y_{N}^{\dagger} Y_{N} Y_{N}^{\dagger}\right) \\
& \left.\left.\left.\left.-\frac{27}{4} \operatorname{Tr}\left(Y_{u} Y_{u}^{\dagger} Y_{u} Y_{u}^{\dagger}\right)\right)\right)+675 g_{2}^{2}-960 \lambda_{1}\right)\right] \\
&
\end{aligned}
$$

Open Access. This article is distributed under the terms of the Creative Commons Attribution License (CC-BY 4.0), which permits any use, distribution and reproduction in any medium, provided the original author(s) and source are credited. 


\section{References}

[1] ATLAS collaboration, Observation of a new particle in the search for the Standard Model Higgs boson with the ATLAS detector at the LHC, Phys. Lett. B 716 (2012) 1 [arXiv:1207.7214] [INSPIRE].

[2] CMS collaboration, Observation of a New Boson at a Mass of 125 GeV with the CMS Experiment at the LHC, Phys. Lett. B 716 (2012) 30 [arXiv:1207.7235] [INSPIRE].

[3] ATLAS collaboration, Evidence for the spin-0 nature of the Higgs boson using ATLAS data, Phys. Lett. B 726 (2013) 120 [arXiv:1307.1432] [INSPIRE].

[4] CMS collaboration, Combined measurements of Higgs boson couplings in proton-proton collisions at $\sqrt{s}=13$ TeV, Eur. Phys. J. C 79 (2019) 421 [arXiv:1809.10733] [InSPIRE].

[5] ATLAS collaboration, Combined measurements of Higgs boson production and decay using up to $80 \mathrm{fb}^{-1}$ of proton-proton collision data at $\sqrt{\mathrm{s}}=13$ TeV collected with the ATLAS experiment, Tech. Rep. ATLAS-CONF-2018-031, CERN, Geneva (Jul, 2018).

[6] G. Isidori, G. Ridolfi and A. Strumia, On the metastability of the standard model vacuum, Nucl. Phys. B 609 (2001) 387 [hep-ph/0104016] [INSPIRE].

[7] F. Bezrukov, M.Y. Kalmykov, B.A. Kniehl and M. Shaposhnikov, Higgs Boson Mass and New Physics, JHEP 10 (2012) 140 [arXiv:1205.2893] [INSPIRE].

[8] G. Degrassi et al., Higgs mass and vacuum stability in the Standard Model at NNLO, JHEP 08 (2012) 098 [arXiv: 1205.6497] [INSPIRE].

[9] D. Buttazzo et al., Investigating the near-criticality of the Higgs boson, JHEP 12 (2013) 089 [arXiv: 1307.3536] [INSPIRE].

[10] M. Gonderinger, H. Lim and M.J. Ramsey-Musolf, Complex Scalar Singlet Dark Matter: Vacuum Stability and Phenomenology, Phys. Rev. D 86 (2012) 043511 [arXiv:1202.1316] [INSPIRE].

[11] M. Gonderinger, Y. Li, H. Patel and M.J. Ramsey-Musolf, Vacuum Stability, Perturbativity, and Scalar Singlet Dark Matter, JHEP 01 (2010) 053 [arXiv:0910.3167] [INSPIRE].

[12] R. Costa, A.P. Morais, M.O.P. Sampaio and R. Santos, Two-loop stability of a complex singlet extended Standard Model, Phys. Rev. D 92 (2015) 025024 [arXiv:1411.4048] [INSPIRE].

[13] N. Haba and Y. Yamaguchi, Vacuum stability in the $\mathrm{U}(1)_{\chi}$ extended model with vanishing scalar potential at the Planck scale, PTEP 2015 (2015) 093B05 [arXiv:1504.05669] [INSPIRE].

[14] W.-L. Guo and Y.-L. Wu, The Real singlet scalar dark matter model, JHEP 10 (2010) 083 [arXiv:1006.2518] [INSPIRE].

[15] V. Barger, P. Langacker, M. McCaskey, M. Ramsey-Musolf and G. Shaughnessy, Complex Singlet Extension of the Standard Model, Phys. Rev. D 79 (2009) 015018 [arXiv:0811.0393] [INSPIRE].

[16] N. Khan and S. Rakshit, Study of electroweak vacuum metastability with a singlet scalar dark matter, Phys. Rev. D 90 (2014) 113008 [arXiv:1407.6015] [InSPIRE].

[17] S. Baek, P. Ko, W.-I. Park and E. Senaha, Vacuum structure and stability of a singlet fermion dark matter model with a singlet scalar messenger, JHEP 11 (2012) 116 [arXiv: 1209.4163] [INSPIRE]. 
[18] A. Datta, A. Elsayed, S. Khalil and A. Moursy, Higgs vacuum stability in the B $-L$ extended standard model, Phys. Rev. D 88 (2013) 053011 [arXiv:1308.0816] [InSPIRE].

[19] J. Chakrabortty, P. Konar and T. Mondal, Constraining a class of B-L extended models from vacuum stability and perturbativity, Phys. Rev. D 89 (2014) 056014 [arXiv:1308.1291] [INSPIRE].

[20] P. Bandyopadhyay and R. Mandal, Vacuum stability in an extended standard model with a leptoquark, Phys. Rev. D 95 (2017) 035007 [arXiv:1609.03561] [InSPIRE].

[21] N. Chakrabarty and B. Mukhopadhyaya, High-scale validity of a two Higgs doublet scenario: metastability included, Eur. Phys. J. C 77 (2017) 153 [arXiv:1603.05883] [InSPIRE].

[22] I. Chakraborty and A. Kundu, Scalar potential of two-Higgs doublet models, Phys. Rev. D 92 (2015) 095023 [arXiv: 1508.00702] [INSPIRE].

[23] P.M. Ferreira, R. Santos and A. Barroso, Stability of the tree-level vacuum in two Higgs doublet models against charge or CP spontaneous violation, Phys. Lett. B 603 (2004) 219 [Erratum ibid. 629 (2005) 114] [hep-ph/0406231] [INSPIRE].

[24] M. Maniatis, A. von Manteuffel, O. Nachtmann and F. Nagel, Stability and symmetry breaking in the general two-Higgs-doublet model, Eur. Phys. J. C 48 (2006) 805 [hep-ph/0605184] [INSPIRE].

[25] A. Barroso, P.M. Ferreira, R. Santos and J.P. Silva, Stability of the normal vacuum in multi-Higgs-doublet models, Phys. Rev. D 74 (2006) 085016 [hep-ph/0608282] [INSPIRE].

[26] R.A. Battye, G.D. Brawn and A. Pilaftsis, Vacuum Topology of the Two Higgs Doublet Model, JHEP 08 (2011) 020 [arXiv:1106.3482] [InSPIRE].

[27] K. Kannike, Vacuum Stability of a General Scalar Potential of a Few Fields, Eur. Phys. J. C 76 (2016) 324 [Erratum ibid. 78 (2018) 355] [arXiv: 1603. 02680] [INSPIRE].

[28] X.-J. Xu, Tree-level vacuum stability of two-Higgs-doublet models and new constraints on the scalar potential, Phys. Rev. D 95 (2017) 115019 [arXiv:1705.08965] [INSPIRE].

[29] N. Haba and Y. Yamaguchi, Vacuum stability in the $\mathrm{U}(1)_{\chi}$ extended model with vanishing scalar potential at the Planck scale, PTEP 2015 (2015) 093B05 [arXiv:1504.05669] [INSPIRE].

[30] S. Oda, N. Okada and D.-s. Takahashi, Classically conformal U(1)' extended standard model and Higgs vacuum stability, Phys. Rev. D 92 (2015) 015026 [arXiv: 1504.06291] [InSPIRE].

[31] A. Das, N. Okada and N. Papapietro, Electroweak vacuum stability in classically conformal B-L extension of the Standard Model, Eur. Phys. J. C 77 (2017) 122 [arXiv:1509.01466] [INSPIRE].

[32] A. Das, S. Oda, N. Okada and D.-s. Takahashi, Classically conformal U(1)' extended standard model, electroweak vacuum stability, and LHC Run-2 bounds, Phys. Rev. D 93 (2016) 115038 [arXiv: 1605.01157] [INSPIRE].

[33] P. Ghosh, A.K. Saha and A. Sil, Study of Electroweak Vacuum Stability from Extended Higgs Portal of Dark Matter and Neutrinos, Phys. Rev. D 97 (2018) 075034 [arXiv:1706.04931] [INSPIRE].

[34] N. Chakrabarty, D.K. Ghosh, B. Mukhopadhyaya and I. Saha, Dark matter, neutrino masses and high scale validity of an inert Higgs doublet model, Phys. Rev. D 92 (2015) 015002 [arXiv: 1501.03700] [INSPIRE]. 
[35] B. Swiezewska, Inert scalars and vacuum metastability around the electroweak scale, JHEP 07 (2015) 118 [arXiv:1503.07078] [INSPIRE].

[36] N. Khan and S. Rakshit, Constraints on inert dark matter from the metastability of the electroweak vacuum, Phys. Rev. D 92 (2015) 055006 [arXiv: 1503.03085] [INSPIRE].

[37] A. Belyaev, G. Cacciapaglia, I.P. Ivanov, F. Rojas-Abatte and M. Thomas, Anatomy of the Inert Two Higgs Doublet Model in the light of the LHC and non-LHC Dark Matter Searches, Phys. Rev. D 97 (2018) 035011 [arXiv: 1612.00511] [INSPIRE].

[38] S. Jangid and P. Bandyopadhyay, Distinguishing Inert Higgs Doublet and Inert Triplet Scenarios, Eur. Phys. J. C 80 (2020) 715 [arXiv:2003.11821] [InSPIRE].

[39] A.D. Plascencia, Classical scale invariance in the inert doublet model, JHEP 09 (2015) 026 [arXiv: 1507.04996] [INSPIRE].

[40] S. Yaser Ayazi and S.M. Firouzabadi, Footprint of Triplet Scalar Dark Matter in Direct, Indirect Search and Invisible Higgs Decay, Cogent Phys. 2 (2015) 1047559 [arXiv: 1501.06176] [INSPIRE].

[41] N. Khan, Exploring the hyperchargeless Higgs triplet model up to the Planck scale, Eur. Phys. J. C 78 (2018) 341 [arXiv:1610.03178] [INSPIRE].

[42] I. Gogoladze, N. Okada and Q. Shafi, Higgs boson mass bounds in a type-II seesaw model with triplet scalars, Phys. Rev. D 78 (2008) 085005 [arXiv:0802.3257] [INSPIRE].

[43] E.J. Chun, H.M. Lee and P. Sharma, Vacuum Stability, Perturbativity, EWPD and Higgs-to-diphoton rate in Type II Seesaw Models, JHEP 11 (2012) 106 [arXiv:1209.1303] [INSPIRE].

[44] P.S. Bhupal Dev, D.K. Ghosh, N. Okada and I. Saha, 125 GeV Higgs Boson and the Type-II Seesaw Model, JHEP 03 (2013) 150 [Erratum ibid. 05 (2013) 049] [arXiv:1301.3453] [INSPIRE].

[45] A. Kobakhidze and A. Spencer-Smith, Neutrino Masses and Higgs Vacuum Stability, JHEP 08 (2013) 036 [arXiv: 1305.7283] [InSPIRE].

[46] C. Bonilla, R.M. Fonseca and J.W.F. Valle, Consistency of the triplet seesaw model revisited, Phys. Rev. D 92 (2015) 075028 [arXiv: 1508.02323] [InSPIRE].

[47] N. Haba, H. Ishida, N. Okada and Y. Yamaguchi, Vacuum stability and naturalness in type-II seesaw, Eur. Phys. J. C 76 (2016) 333 [arXiv:1601.05217] [InSPIRE].

[48] P.S.B. Dev, C.M. Vila and W. Rodejohann, Naturalness in testable type-II seesaw scenarios, Nucl. Phys. B 921 (2017) 436 [arXiv:1703.00828] [InSPIRE].

[49] R.N. Mohapatra, Limits on the Mass of the Right-handed Majorana Neutrino, Phys. Rev. D 34 (1986) 909 [INSPIRE].

[50] P.S. Bhupal Dev, R.N. Mohapatra, W. Rodejohann and X.-J. Xu, Vacuum structure of the left-right symmetric model, JHEP 02 (2019) 154 [arXiv:1811.06869] [INSPIRE].

[51] G. Chauhan, Vacuum Stability and Symmetry Breaking in Left-Right Symmetric Model, JHEP 12 (2019) 137 [arXiv:1907.07153] [INSPIRE].

[52] C. Cai, Z.-M. Huang, Z. Kang, Z.-H. Yu and H.-H. Zhang, Perturbativity Limits for Scalar Minimal Dark Matter with Yukawa Interactions: Septuplet, Phys. Rev. D 92 (2015) 115004 [arXiv: 1510.01559] [INSPIRE].

[53] C. Cai, Z. Kang, Z. Luo, Z.-H. Yu and H.-H. Zhang, Scalar quintuplet minimal dark matter with Yukawa interactions: perturbative up to the Planck scale, Chin. Phys. C 43 (2019) 023102 [arXiv: 1711.07396] [INSPIRE]. 
[54] S. Jangid, P. Bandyopadhyay, P.S. Bhupal Dev and A. Kumar, Vacuum stability in inert Higgs doublet model with right-handed neutrinos, JHEP 08 (2020) 154 [arXiv:2001.01764] [INSPIRE].

[55] S. Ipek, A.D. Plascencia and J. Turner, Assessing Perturbativity and Vacuum Stability in High-Scale Leptogenesis, JHEP 12 (2018) 111 [arXiv:1806.00460] [INSPIRE].

[56] C. Corianò, L. Delle Rose and C. Marzo, Vacuum Stability in U(1)' Extensions of the Standard Model with TeV Scale Right Handed Neutrinos, Phys. Lett. B 738 (2014) 13 [arXiv: 1407.8539] [INSPIRE].

[57] C. Corianò, L. Delle Rose and C. Marzo, Constraints on abelian extensions of the Standard Model from two-loop vacuum stability and $\mathrm{U}(1)_{B-L}$, JHEP 02 (2016) 135 [arXiv: 1510.02379] [INSPIRE].

[58] L. Delle Rose, C. Marzo and A. Urbano, On the stability of the electroweak vacuum in the presence of low-scale seesaw models, JHEP 12 (2015) 050 [arXiv:1506.03360] [INSPIRE].

[59] I. Garg, S. Goswami, K.N. Vishnudath and N. Khan, Electroweak vacuum stability in presence of singlet scalar dark matter in TeV scale seesaw models, Phys. Rev. D 96 (2017) 055020 [arXiv: 1706.08851] [INSPIRE].

[60] J.A. Casas, V. Di Clemente, A. Ibarra and M. Quirós, Massive neutrinos and the Higgs mass window, Phys. Rev. D 62 (2000) 053005 [hep-ph/9904295] [INSPIRE].

[61] J. Elias-Miro, J.R. Espinosa, G.F. Giudice, G. Isidori, A. Riotto and A. Strumia, Higgs mass implications on the stability of the electroweak vacuum, Phys. Lett. B $\mathbf{7 0 9}$ (2012) 222 [arXiv: 1112.3022] [INSPIRE].

[62] W. Rodejohann and H. Zhang, Impact of massive neutrinos on the Higgs self-coupling and electroweak vacuum stability, JHEP 06 (2012) 022 [arXiv:1203.3825] [INSPIRE].

[63] I. Masina, Higgs boson and top quark masses as tests of electroweak vacuum stability, Phys. Rev. D 87 (2013) 053001 [arXiv: 1209.0393] [INSPIRE].

[64] M. Farina, D. Pappadopulo and A. Strumia, A modified naturalness principle and its experimental tests, JHEP 08 (2013) 022 [arXiv: 1303.7244] [INSPIRE].

[65] J.N. Ng and A. de la Puente, Electroweak Vacuum Stability and the Seesaw Mechanism Revisited, Eur. Phys. J. C 76 (2016) 122 [arXiv:1510.00742] [InSPIRE].

[66] G. Bambhaniya, P.S. Bhupal Dev, S. Goswami, S. Khan and W. Rodejohann, Naturalness, Vacuum Stability and Leptogenesis in the Minimal Seesaw Model, Phys. Rev. D 95 (2017) 095016 [arXiv: 1611.03827] [INSPIRE].

[67] S. Khan, S. Goswami and S. Roy, Vacuum Stability constraints on the minimal singlet TeV Seesaw Model, Phys. Rev. D 89 (2014) 073021 [arXiv: 1212.3694] [InSPIRE].

[68] A. Das, S. Goswami, K.N. Vishnudath and T. Nomura, Constraining a general U(1)' inverse seesaw model from vacuum stability, dark matter and collider, Phys. Rev. D 101 (2020) 055026 [arXiv : 1905.00201] [INSPIRE].

[69] S. Baek, P. Ko, W.-I. Park and E. Senaha, Vacuum structure and stability of a singlet fermion dark matter model with a singlet scalar messenger, JHEP 11 (2012) 116 [arXiv: 1209.4163] [INSPIRE].

[70] M. Lindner, M. Platscher, C.E. Yaguna and A. Merle, Fermionic WIMPs and vacuum stability in the scotogenic model, Phys. Rev. D 94 (2016) 115027 [arXiv:1608.00577] [INSPIRE]. 
[71] A. Dutta Banik, A.K. Saha and A. Sil, Scalar assisted singlet doublet fermion dark matter model and electroweak vacuum stability, Phys. Rev. D 98 (2018) 075013 [arXiv: 1806.08080] [INSPIRE].

[72] J.-W. Wang, X.-J. Bi, P.-F. Yin and Z.-H. Yu, Impact of Fermionic Electroweak Multiplet Dark Matter on Vacuum Stability with One-loop Matching, Phys. Rev. D 99 (2019) 055009 [arXiv: 1811.08743] [INSPIRE].

[73] M.-L. Xiao and J.-H. Yu, Stabilizing electroweak vacuum in a vectorlike fermion model, Phys. Rev. D 90 (2014) 014007 [Addendum ibid. 90 (2014) 019901] [arXiv:1404.0681] [INSPIRE].

[74] S. Gopalakrishna and A. Velusamy, Higgs vacuum stability with vectorlike fermions, Phys. Rev. D 99 (2019) 115020 [arXiv:1812.11303] [INSPIRE].

[75] R.N. Mohapatra and Y. Zhang, TeV Scale Universal Seesaw, Vacuum Stability and Heavy Higgs, JHEP 06 (2014) 072 [arXiv: 1401.6701] [INSPIRE].

[76] P.S.B. Dev, R.N. Mohapatra and Y. Zhang, Quark Seesaw, Vectorlike Fermions and Diphoton Excess, JHEP 02 (2016) 186 [arXiv:1512.08507] [INSPIRE].

[77] Particle Data Group collaboration, Review of Particle Physics, Phys. Rev. D 98 (2018) 030001 [INSPIRE].

[78] T. Markkanen, A. Rajantie and S. Stopyra, Cosmological Aspects of Higgs Vacuum Metastability, Front. Astron. Space Sci. 5 (2018) 40 [arXiv:1809.06923] [INSPIRE].

[79] J.R. Espinosa, Implications of the top (and Higgs) mass for vacuum stability, PoS TOP2015 (2016) 043 [arXiv: 1512.01222] [INSPIRE].

[80] R. Franceschini, T. Hambye and A. Strumia, Type-III see-saw at LHC, Phys. Rev. D 78 (2008) 033002 [arXiv: 0805.1613] [INSPIRE].

[81] R. Foot, H. Lew, X.G. He and G.C. Joshi, Seesaw Neutrino Masses Induced by a Triplet of Leptons, Z. Phys. C 44 (1989) 441 [inSPIRE].

[82] P. Bandyopadhyay and E.J. Chun, Displaced Higgs production in type-III seesaw, JHEP 11 (2010) 006 [arXiv: 1007.2281] [InSPIRE].

[83] P. Bandyopadhyay, S. Choi, E.J. Chun and K. Min, Probing Higgs bosons via the type-III seesaw mechanism at the LHC, Phys. Rev. D 85 (2012) 073013 [arXiv:1112.3080] [INSPIRE].

[84] P. Bandyopadhyay, S. Choubey and M. Mitra, Two Higgs Doublet Type III Seesaw with mu-tau symmetry at LHC, JHEP 10 (2009) 012 [arXiv:0906.5330] [INSPIRE].

[85] R. Ruiz, QCD Corrections to Pair Production of Type III Seesaw Leptons at Hadron Colliders, JHEP 12 (2015) 165 [arXiv:1509.05416] [inSPIRE].

[86] A. Das and S. Mandal, Bounds on the triplet fermions in type-III seesaw and implications for collider searches, arXiv:2006.04123 [INSPIRE].

[87] A. Das, S. Mandal and T. Modak, Testing triplet fermions at the electron-positron and electron-proton colliders using fat jet signatures, Phys. Rev. D 102 (2020) 033001 [arXiv: 2005.02267] [INSPIRE].

[88] S. Jana, N. Okada and D. Raut, Displaced Vertex and Disappearing Track Signatures in type-III Seesaw, arXiv: 1911.09037 [INSPIRE].

[89] M. Mitra, S. Niyogi and M. Spannowsky, Type-II Seesaw Model and Multilepton Signatures at Hadron Colliders, Phys. Rev. D 95 (2017) 035042 [arXiv:1611.09594] [INSPIRE]. 
[90] S.K. Garg, D. Goswami and P. Poulose, Probing the type-III seesaw model through $e^{+} e^{-} \rightarrow \Sigma^{+} \Sigma^{-}$at ILC, J. Phys. Conf. Ser. 481 (2014) 012018 [InSPIRE].

[91] P. Bandyopadhyay, S. Dutta and M. Jakkapu, Exploring angular distributions at the LHC, arXiv: 2007.12997 [INSPIRE].

[92] I. Gogoladze, N. Okada and Q. Shafi, Higgs Boson Mass Bounds in the Standard Model with Type III and Type I Seesaw, Phys. Lett. B 668 (2008) 121 [arXiv:0805.2129] [INSPIRE].

[93] C.-S. Chen and Y. Tang, Vacuum stability, neutrinos, and dark matter, JHEP 04 (2012) 019 [arXiv: 1202.5717] [INSPIRE].

[94] M. Lindner, H.H. Patel and B. Radovčić, Electroweak Absolute, Meta-, and Thermal Stability in Neutrino Mass Models, Phys. Rev. D 93 (2016) 073005 [arXiv:1511.06215] [InSPIRE].

[95] S. Goswami, K.N. Vishnudath and N. Khan, Constraining the minimal type-III seesaw model with naturalness, lepton flavor violation, and electroweak vacuum stability, Phys. Rev. D 99 (2019) 075012 [arXiv: 1810.11687] [INSPIRE].

[96] S. Choubey and A. Kumar, Inflation and Dark Matter in the Inert Doublet Model, JHEP 11 (2017) 080 [arXiv: 1707.06587] [INSPIRE].

[97] A. Goudelis, B. Herrmann and O. Stål, Dark matter in the Inert Doublet Model after the discovery of a Higgs-like boson at the LHC, JHEP 09 (2013) 106 [arXiv:1303.3010] [INSPIRE].

[98] L. Lopez Honorez, Scalar dark matter: A revision of the inert doublet model, Nuovo Cim. C 035N1 (2012) 39 [INSPIRE].

[99] M.H.G. Tytgat, The Inert Doublet Model: A New archetype of WIMP dark matter?, J. Phys. Conf. Ser. 120 (2008) 042026 [arXiv:0712 .4206] [InSPIRE].

[100] L. Lopez Honorez, Dark Matter from the Inert Doublet Model, in 42nd Rencontres de Moriond on Electroweak Interactions and Unified Theories, La Thuile Italy (2007), pg. 277 [arXiv:0706.0186] [INSPIRE].

[101] E.M. Dolle and S. Su, The Inert Dark Matter, Phys. Rev. D 80 (2009) 055012 [arXiv: 0906.1609] [INSPIRE].

[102] L. Lopez Honorez and C.E. Yaguna, The inert doublet model of dark matter revisited, JHEP 09 (2010) 046 [arXiv: 1003.3125] [INSPIRE].

[103] L. Lopez Honorez and C.E. Yaguna, A new viable region of the inert doublet model, JCAP 01 (2011) 002 [arXiv: 1011.1411] [INSPIRE].

[104] A. Arhrib, Y.-L.S. Tsai, Q. Yuan and T.-C. Yuan, An Updated Analysis of Inert Higgs Doublet Model in light of the Recent Results from LUX, PLANCK, AMS-02 and LHC, JCAP 06 (2014) 030 [arXiv:1310.0358] [INSPIRE].

[105] L. Lopez Honorez, E. Nezri, J.F. Oliver and M.H.G. Tytgat, The Inert Doublet Model: An Archetype for Dark Matter, JCAP 02 (2007) 028 [hep-ph/0612275] [INSPIRE].

[106] M. Cirelli, N. Fornengo and A. Strumia, Minimal dark matter, Nucl. Phys. B 753 (2006) 178 [hep-ph/0512090] [INSPIRE].

[107] S. Banerjee, F. Boudjema, N. Chakrabarty, G. Chalons and H. Sun, Relic density of dark matter in the inert doublet model beyond leading order: The heavy mass case, Phys. Rev. D 100 (2019) 095024 [arXiv: 1906.11269] [INSPIRE].

[108] G.C. Branco, P.M. Ferreira, L. Lavoura, M.N. Rebelo, M. Sher and J.P. Silva, Theory and phenomenology of two-Higgs-doublet models, Phys. Rept. 516 (2012) 1 [arXiv:1106.0034] [INSPIRE]. 
[109] A.D. Plascencia, Classical scale invariance in the inert doublet model, JHEP 09 (2015) 026 [arXiv: 1507.04996] [INSPIRE].

[110] A. Atre, T. Han, S. Pascoli and B. Zhang, The Search for Heavy Majorana Neutrinos, JHEP 05 (2009) 030 [arXiv:0901.3589] [inSPIRE].

[111] F.F. Deppisch, P.S. Bhupal Dev and A. Pilaftsis, Neutrinos and Collider Physics, New J. Phys. 17 (2015) 075019 [arXiv: 1502.06541] [INSPIRE].

[112] F. del Aguila, J. de Blas and M. Pérez-Victoria, Effects of new leptons in Electroweak Precision Data, Phys. Rev. D 78 (2008) 013010 [arXiv:0803.4008] [INSPIRE].

[113] E. Akhmedov, A. Kartavtsev, M. Lindner, L. Michaels and J. Smirnov, Improving Electro-Weak Fits with TeV-scale Sterile Neutrinos, JHEP 05 (2013) 081 [arXiv: 1302.1872] [INSPIRE].

[114] J. de Blas, Electroweak limits on physics beyond the Standard Model, EPJ Web Conf. 60 (2013) 19008 [arXiv: 1307.6173] [INSPIRE].

[115] R.N. Mohapatra, Mechanism for Understanding Small Neutrino Mass in Superstring Theories, Phys. Rev. Lett. 56 (1986) 561 [INSPIRE].

[116] R.N. Mohapatra and J.W.F. Valle, Neutrino Mass and Baryon Number Nonconservation in Superstring Models, Phys. Rev. D 34 (1986) 1642 [InSPIRE].

[117] P. Bandyopadhyay, E.J. Chun, H. Okada and J.-C. Park, Higgs Signatures in Inverse Seesaw Model at the LHC, JHEP 01 (2013) 079 [arXiv: 1209.4803] [INSPIRE].

[118] P. Bandyopadhyay, E.J. Chun and R. Mandal, Phenomenology of Higgs bosons in inverse seesaw model with Type-X two Higgs doublet at the LHC, JHEP 08 (2019) 169 [arXiv: 1904.09494] [INSPIRE].

[119] S. Ipek, A.D. Plascencia and J. Turner, Assessing Perturbativity and Vacuum Stability in High-Scale Leptogenesis, JHEP 12 (2018) 111 [arXiv:1806.00460] [INSPIRE].

[120] F. Staub, SARAH 4: A tool for (not only SUSY) model builders, Comput. Phys. Commun. 185 (2014) 1773 [arXiv: 1309.7223] [INSPIRE].

[121] S.R. Coleman and E.J. Weinberg, Radiative Corrections as the Origin of Spontaneous Symmetry Breaking, Phys. Rev. D 7 (1973) 1888 [InSPIRE].

[122] J.A. Casas, J.R. Espinosa, M. Quirós and A. Riotto, The Lightest Higgs boson mass in the minimal supersymmetric standard model, Nucl. Phys. B 436 (1995) 3 [Erratum ibid. 439 (1995) 466] [hep-ph/9407389] [INSPIRE].

[123] B. Eiteneuer, A. Goudelis and J. Heisig, The inert doublet model in the light of Fermi-LAT gamma-ray data: a global fit analysis, Eur. Phys. J. C 77 (2017) 624 [arXiv:1705.01458] [INSPIRE].

[124] M.A. Díaz, B. Koch and S. Urrutia-Quiroga, Constraints to Dark Matter from Inert Higgs Doublet Model, Adv. High Energy Phys. 2016 (2016) 8278375 [arXiv:1511.04429] [INSPIRE].

[125] C. Garcia-Cely and A. Ibarra, Signatures of the Inert Doublet Dark Matter Model, Nucl. Part. Phys. Proc. 263-264 (2015) 107 [inSPIRE].

[126] S. Bhattacharya, P. Ghosh, A.K. Saha and A. Sil, Two component dark matter with inert Higgs doublet: neutrino mass, high scale validity and collider searches, JHEP 03 (2020) 090 [arXiv: 1905.12583] [INSPIRE]. 
[127] J. Kopp, Constraints on dark matter annihilation from AMS-02 results, Phys. Rev. D 88 (2013) 076013 [arXiv: 1304.1184] [INSPIRE].

[128] P. Bandyopadhyay and A. Costantini, Obscure Higgs boson at Colliders, Phys. Rev. D 103 (2021) 015025 [arXiv: 2010.02597] [INSPIRE].

[129] P. Bandyopadhyay, K. Huitu and S. Niyogi, Non-standard charged Higgs decay at the LHC in Next-to-Minimal Supersymmetric Standard Model, JHEP 07 (2016) 015 [arXiv: 1512.09241] [INSPIRE].

[130] P. Bandyopadhyay, C. Corianò and A. Costantini, General analysis of the charged Higgs sector of the $Y=0$ triplet-singlet extension of the MSSM at the LHC, Phys. Rev. D 94 (2016) 055030 [arXiv: 1512.08651] [INSPIRE].

[131] P. Bandyopadhyay, K. Huitu and A. Sabanci Keceli, Multi-Lepton Signatures of the Triplet Like Charged Higgs at the LHC, JHEP 05 (2015) 026 [arXiv: 1412.7359] [INSPIRE].

[132] P. Bandyopadhyay, E.J. Chun and J.-C. Park, Right-handed sneutrino dark matter in U(1)' seesaw models and its signatures at the LHC, JHEP 06 (2011) 129 [arXiv:1105.1652] [INSPIRE].

[133] P. Bandyopadhyay, E.J. Chun and R. Mandal, Implications of right-handed neutrinos in B - L extended standard model with scalar dark matter, Phys. Rev. D 97 (2018) 015001 [arXiv: 1707.00874] [INSPIRE].

[134] C.-W. Chiang, G. Cottin, A. Das and S. Mandal, Displaced heavy neutrinos from $Z^{\prime}$ decays at the LHC, JHEP 12 (2019) 070 [arXiv:1908.09838] [INSPIRE].

[135] P. Bandyopadhyay and E.J. Chun, Lepton flavour violating signature in supersymmetric $U(1)^{\prime}$ seesaw models at the LHC, JHEP 05 (2015) 045 [arXiv:1412.7312] [INSPIRE].

[136] P. Bandyopadhyay, Displaced lepton flavour violating signatures of right-handed sneutrinos in $U(1)^{\prime}$ supersymmetric models, JHEP 09 (2017) 052 [arXiv: 1511.03842] [INSPIRE].

[137] S. Jana, N. Okada and D. Raut, Displaced vertex signature of type-I seesaw model, Phys. Rev. D 98 (2018) 035023 [arXiv:1804.06828] [INSPIRE].

[138] S. Jana, N. Okada and D. Raut, Displaced Vertex and Disappearing Track Signatures in type-III Seesaw, arXiv:1911.09037 [INSPIRE].

[139] P. Bandyopadhyay, S. Dutta, A. KT and C. Sen, Displaced Higgs production in Type-III Seesaw at the LHC/FCC, MATHUSLA and Muon collider, in preparation.

[140] CMS collaboration, Search for Evidence of the Type-III Seesaw Mechanism in Multilepton Final States in Proton-Proton Collisions at $\sqrt{s}=13 \mathrm{TeV}$, Phys. Rev. Lett. 119 (2017) 221802 [arXiv: 1708.07962] [INSPIRE]. 